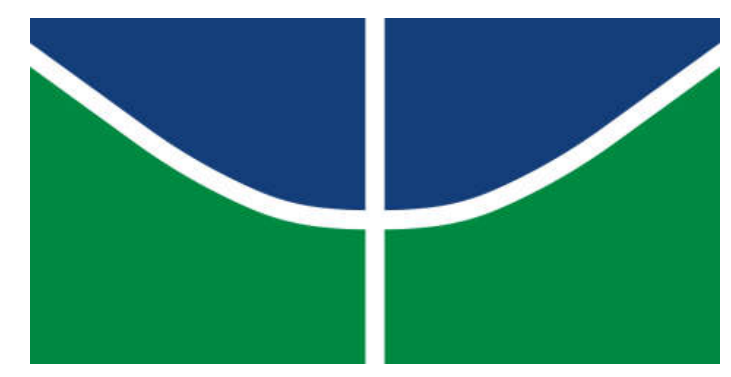

UNIVERSIDADE DE BRASÍLIA - UnB

FACULDADE DE AGRONOMIA E MEDICINA VETERINÁRIA - FAV

PROPOSIÇÃO DE UM MODELO DE SENSOR DE UMIDADE DO SOLO PELO PRINCÍPIO DE TRANSFERÊNCIA DE CALOR

ISAC JEFERSON FERREIRA DE SOUSA 
ISAC JEFERSON FERREIRA DE SOUSA

PROPOSIÇÃO DE UM MODELO DE SENSOR DE UMIDADE DO SOLO PELO PRINCÍPIO DE TRANSFERÊNCIA DE CALOR

Trabalho de conclusão de curso apresentado à Faculdade de Agronomia e Medicina Veterinária como exigência final para obtenção do título de Engenheiro Agrônomo. Orientador: Prof. Dr. Tairone Paiva Leão

Brasília 
Sousa, Isac Jeferson Ferreira.

\section{PROPOSIÇÃO DE UM MODELO DE SENSOR DE UMIDADE DO SOLO PELO PRINCÍPIO DE TRANSFERÊNCIA DE CALOR/}

Isac Jeferson Ferreira de Sousa. Orientação: Tairone Paiva Leão, Brasília, 2019.

Monografia - Universidade de Brasília / Faculdade de Agronomia e Medicina Veterinária, 2019.

40 p. : il.

1. Umidade do solo. 2. Pulso de calor. 3. Sensor de baixo custo.

I. LEÃO, T.P. II. Ph. D.

REFERÊNCIA BIBLIOGRÁFICA

SOUSA, I. J. F. PROPOSIÇÃO DE UM MODELO DE SENSOR DE UMIDADE DO SOLO PELO PRINCÍPIO DE TRANSFERÊNCIA DE CALOR. Brasília: Faculdade de Agronomia e Medicina Veterinária, Universidade de Brasília, 2019. 40 p. Monografia.

\section{CESSÃO DE DIREITOS}

Nome do Autor: ISAC JEFERSON FERREIRA DE SOUSA

Título da Monografia de Conclusão de Curso: Proposição de um modelo de sensor de umidade do solo pelo princípio de transferência de calor.

Grau: $3^{\circ}$

Ano: 2019

É concedida à Universidade de Brasília permissão para reproduzir cópias desta monografia de graduação e para emprestar ou vender tais cópias somente para propósitos acadêmicos e científicos. O autor reserva-se a outros direitos de publicação e nenhuma parte desta monografia de graduação pode ser reproduzida sem autorização por escrito do autor.

Isac Jeferson Ferreira de Sousa

CPF: $x x x . x x x . x x x-x x$

Endereço: Universidade de Brasília - Campus Darcy Ribeiro, Asa Norte.

CEP: 70910-900. Brasília, DF. Brasil 
PROPOSIÇÃO DE UM MODELO DE SENSOR DE UMIDADE DO SOLO PELO PRINCÍPIO DE TRANSFERÊNCIA DE CALOR

ISAC JEFERSON FERREIRA DE SOUSA

Trabalho de conclusão de curso submetido à Faculdade de Agronomia e Medicina Veterinária - FAV da Universidade de Brasília - UnB, para obtenção do grau de Engenheiro Agrônomo.

APROVADO PELA BANCA EXAMINADORA EM:

BANCA EXAMINADORA:

TAIRONE PAIVA LEAO

Professor da Faculdade de Agronomia e Medicina Veterinária, FAV-UnB. (ORIENTADOR)

DELVIO SANDRI

Professor da Faculdade de Agronomia e Medicina Veterinária, FAV-UnB. (EXAMINADOR)

RODRIGO MOURA PEREIRA

Engenheiro Agrícola, Doutorando em Agronomia, FAV-UnB.

(EXAMINADOR) 


\section{AGRADECIMENTOS}

Gostaria de expressar meus agradecimentos a todos que fizeram parte da minha vida durante o ensino superior. Em especial aos meus pais que sempre me deram todo apoio possível.

Gostaria de agradecer também a minha tia Cássia e ao meu irmão Warlisson que me deram toda base para que eu não tivesse outras preocupações fora da UnB, o apoio de vocês foi fundamental.

Também sou grato pela UnB e por toda ajuda e oportunidades que recebi, agradeço aos Professores Delvio, Tairone e ao Rodrigo por acreditarem no meu potencial e terem me permitido trabalhar na minha área de interesse.

Aos meus amigos Ana, Dudu, Nat, Wanessa e Beto, que me ajudaram a manter a saúde mental durante todos esses anos de estudo, e a Bruna que sempre aparecia para salvar meu semestre nas matérias difíceis.

Sou grato também por ter conhecido minha namorada, a quem devo muito das coisas boas que me aconteceram. 


\section{RESUMO}

O intenso processo de modernização nos diversos setores da economia nos últimos anos, tem criado um processo desigualdade na agricultura, onde os agricultores que não possuem recursos financeiros para adquirir tecnologias, acabam prejudicados em termos de competitividade. O objetivo deste trabalho é propor um sensor de baixo custo para fazer o monitoramento da umidade do solo, auxiliando assim uma maior e melhor implementação de uma irrigação localizada e de precisão. O sensor construído utiliza o princípio da transferência de calor. O solo como um meio poroso, tem seu coeficiente térmico é alterado em função do material que preenche os poros. O sensor proposto é constituído por dois resistores de $2 \mathrm{~W}$ que emitem calor por tempo fixo e de um termistor que detecta a alteração de temperatura causada pela fonte de calor. A propagação desse calor no meio é alterada devido ao material que preenche os poros do solo, sendo a água um dos principais fatores que causam essa alteração. Dessa forma é possível determinar a quantidade de água que se tem no solo ao conhecer os valores do coeficiente térmico dos materiais minerais que o compõem. Avaliações feitas com amostras de areia peneirada e seca em estufa, apresentaram resultados coerentes e promissores. Foi ainda detectada uma redução na precisão de leitura quando a umidade está acima de $60 \%$ da capacidade de campo o que criou a necessidade de novos experimentos.

Palavras-chave: Agricultura de precisão, sensores, umidade do solo, irrigação. 


\begin{abstract}
The intense process of modernization in the various sectors of the economy in recent years has created a process of inequality in agriculture, where farmers who do not have the financial resources to acquire technologies end up harmed in terms of competitiveness. The objective of this work is to propose a low-cost sensor to monitor soil moisture, thus assisting a greater and better implementation of localized and precision irrigation. The constructed sensor uses the principle of heat transfer. The soil as a porous medium, its thermal coefficient is changed depending on the material that fills the pores. The proposed sensor consists of two $2 \mathrm{~W}$ resistors that emit heat for a fixed time and a thermistor that detects the temperature change caused by the heat source. The propagation of this heat in the medium is altered due to the material that fills the pores of the soil, being water one of the main factors that cause this change. Thus, it is possible to determine the amount of water in the soil by knowing the values of the thermal coefficient of the mineral materials that compose it. Evaluations made with sieved and kiln-dried sand samples showed coherent and promising results. A reduction in reading accuracy was detected when humidity is above $60 \%$ of field capacity which created the need for further experiments.
\end{abstract}

Keywords: Precision agriculture, sensors, soil moisture, irrigation. 


\section{SUMÁRIO}

1 INTRODUÇÃO

2 OBJETIVOS

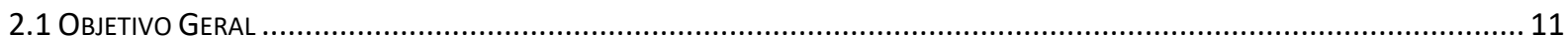

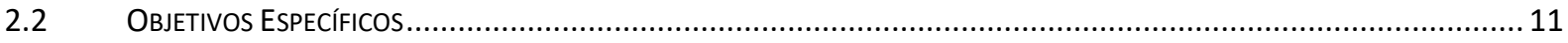

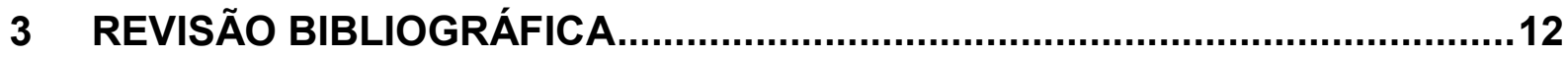

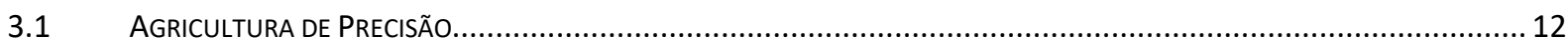

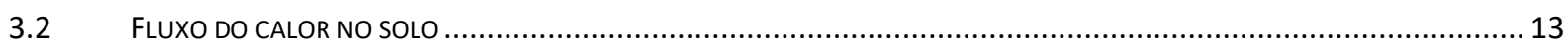

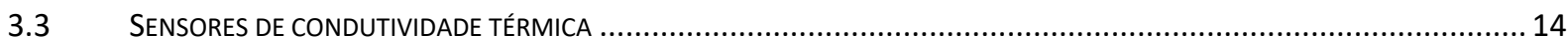

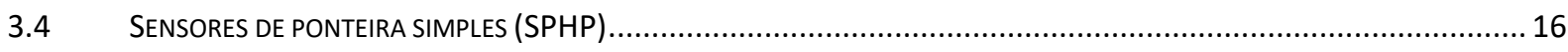

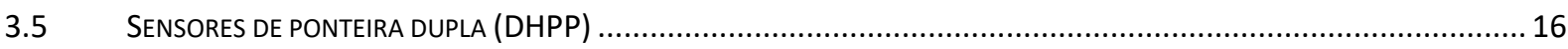

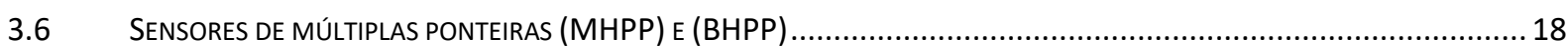

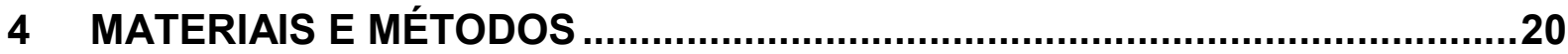

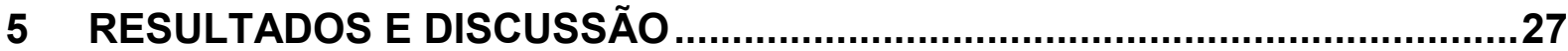

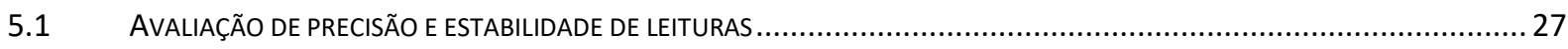

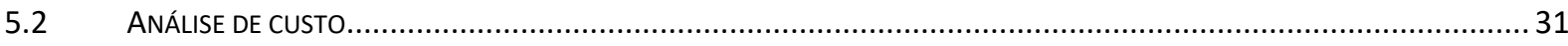

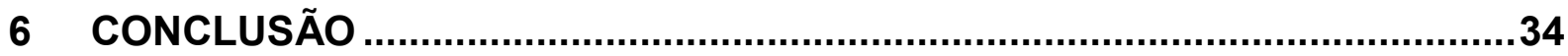

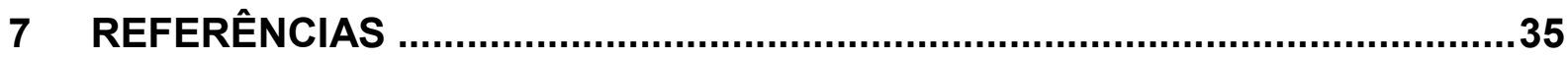

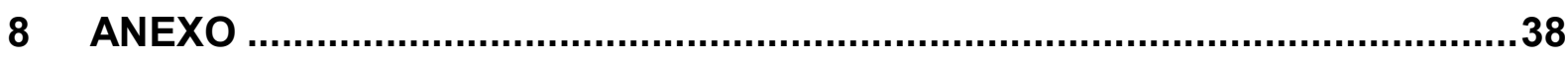




\section{INTRODUÇÃO}

Um dos grandes desafios da agricultura atualmente é conciliar o aumento da produção com a preservação dos recursos, ou seja, crescer de maneira sustentável. Para que isso ocorra, um dos pontos a serem melhorados, segundo Embrapa (2019), é a otimização do uso da água sendo a agricultura de precisão uma possível ferramenta para atingir esse objetivo.

A implementação de recursos tecnológicos possibilita a otimização do uso da água e outros insumos, monitorando em tempo real a necessidade hídrica das plantas e evitando que a mesma quantidade seja aplicada em toda a área, aplicando assim somente o que é necessário em cada parcela.

Um dos principais pontos a serem abordados quanto a irrigação é a correta mensuração da umidade do solo para assim se definir o quanto se deve irrigar. Para isso já foram desenvolvidos diversos tipos de sensores capazes de medir direta ou indiretamente a quantidade de água no solo e com diferentes princípios de funcionamento, como sensores de nêutrons, eletromagnéticos, tensiométricos, capacitivos, resistivos, dentre outros. A maioria desses sensores utilizam princípios indiretos parar medir a umidade do solo, já que uma medida direta além de complexa tem um alto custo o que poderia inviabilizar o uso em campo (GREACEN, 1981; BITELLA et al, 2014; FRANCA, 2015).

Os sensores de umidade do solo baseados em pulso térmico medem indiretamente a umidade a partir do princípio da transferência de calor, com base nas propriedades térmicas dos materiais porosos, onde esses têm seu coeficiente térmico alterado em função do conteúdo de água nos poros (CARSON, 2015). No caso do sensor de fluxo térmico é medida a alteração da capacidade térmica do solo quando os poros estão preenchidos por uma determinada quantidade de água.

Dentro dos sensores de fluxo térmico existem diversos modelos que determinam a umidade a partir da influência da água na capacidade de transferência de calor do solo, todos eles se baseiam no mesmo princípio de montagem onde uma resistência normalmente construída de Níquel-Cromo ( $\mathrm{NiCr}$ ) converte uma corrente elétrica em calor, que é irradiado em todas as direções, enquanto um sensor de temperatura que fica a uma distância conhecida da fonte de calor registra as alterações de temperatura (BRISTOW et al., 1994) 
O uso de um sensor de umidade do solo que possa quantificar a umidade do solo é importante para que se possa reduzir o uso de água no campo. Ao mesmo tempo, é necessário que este não tenha um alto custo, o que poderia inviabilizar seu uso, já que existe uma grande resistência dos produtores para adotarem tecnologias que tenham alto custo e com retorno de longo prazo (MACHADO et al., 2018). Nesse trabalho é apresentado um sensor com potencial para preencher essas necessidades, ou seja, que possa ser de baixo custo, fácil utilização e com precisão aceitável para uma agricultura tecnificada. 


\section{OBJETIVOS}

\subsection{Objetivo Geral}

Desenvolver e avaliar um modelo de sensor térmico de umidade do solo pelo princípio de transferência de calor.

\subsection{Objetivos Específicos}

i) Utilizar materiais e técnicas de fácil acesso e reprodução.

ii) Determinar a faixa de trabalho dos sensores e sua precisão. 


\section{REVISÃO BIBLIOGRÁFICA}

\subsection{Agricultura de Precisão}

Criado a cerca de 25 anos atrás o termo Agricultura de Precisão (AP) se refere em parte ao princípio da regionalização das variáveis campo, de forma que o solo não sendo uniforme em grandes áreas, não deve ser tratado como tal. Assim é necessário que técnicas sejam aplicadas para levar em consideração a variabilidade espacial do solo e assim a diferenciação do tipo e da quantidade de insumos que devem ser aplicados ali para uma melhor performance da produção. Segundo Molin (2015) a definição de AP não é um consenso, podendo variar dependendo do foco de estudo em cada área, a Comissão Brasileira de Agricultura de precisão define a AP como um "conjunto de ferramentas e tecnologias aplicadas para permitir um sistema de gerenciamento agrícola baseado na variabilidade espacial e temporal da unidade produtiva, visando ao aumento de retorno econômico e a redução do impacto ambiental" (Brasil, 2014, p. 6).

E notável observar que praticamente todo o foco da AP está direcionado ao uso de insumos de maneira que tem sido grande a implementação de equipamentos agrícolas que visam principalmente a aplicação variada de adubo baseado no tipo de solo e necessidade da planta (Figura 1).

A ausência de sensoriamento de umidade do solo se dá em parte devido à dificuldade de implementação desses sensores e pelo fato de que, em campo, existem métodos confiáveis de verificar a necessidade de água a ser aplicada. No caso o cálculo da necessidade de irrigação se baseia na evapotranspiração das culturas, pode-se citar o método de Hargreaves e Samani (1985) pela simplicidade de aplicação, pois necessitam apenas dos dados de temperatura e o método de PenmanMonteith FAO (ALLEN et al., 1998), mais utilizado por sua maior abrangência e precisão. 


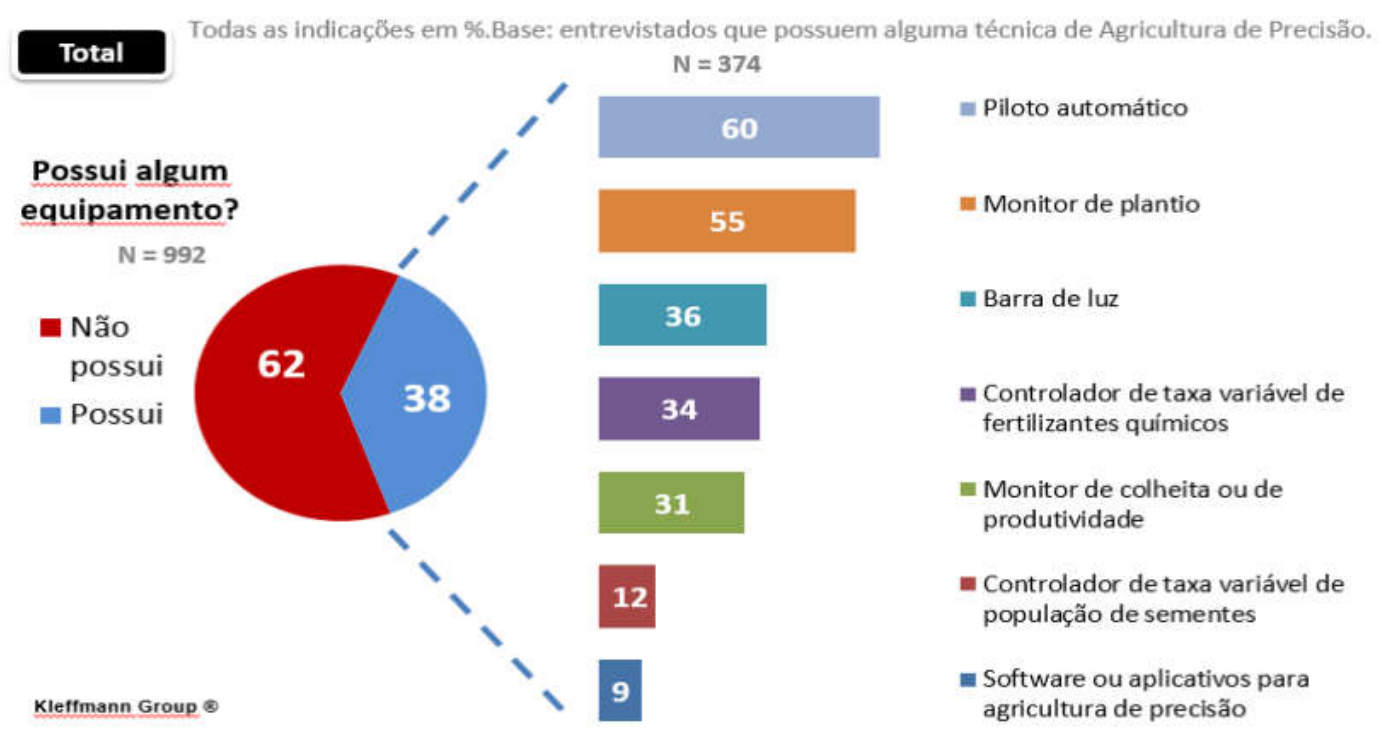

Figura 1. Frequência de adoção de tecnologias para o monitoramento das operações de automação das máquinas (MOLIN, 2017).

\subsection{Fluxo do calor no solo}

A transferência de calor no solo pode ocorrer principalmente de três formas diferentes, por radiação eletromagnética, convecção e condução (HILLEL, 1998). A radiação eletromagnética ocorre quando há agitação das moléculas e isso ocorre em todo corpo com temperatura acima do zero absoluto (0 Kelvin) não dependendo de um meio para que haja a propagação (KITTEL e KROEMER, 2000).

Convecção é o processo de troca de calor que ocorre devido ao movimento de um fluido em um meio, o movimento do fluido facilita a transferência de calor uma vez que e capaz de transferir o calor diretamente ao se mover no ambiente ou pela porosidade do material em que se encontra (CENGEL, 2007).

Assim como a convecção a condução térmica também necessita de um meio para que possa transferir calor, porém não necessita do movimento dos fluidos, no caso da convecção a agitação das moléculas é transferida pela interação entre partículas onde a partícula com maior vibração transfere energia para de menor energia, podendo ocorrer em sólidos e fluídos, até entrarem em equilíbrio (CENGEL, 2007).

Embora o a transferência de calor no solo ocorra por esses três processos o mais significativo é a condução térmica, por isso é preciso se conhecer os componentes do solo, uma vez que materiais diferentes costumam ter capacidades 
diferentes de transferir calor e a alteração no teor desses materiais podem alterar a capacidade de transferência de calor por condução no solo (HILLEL, 1998).

A matéria sólida que forma o solo é preenchida em seus poros por ar ou água de forma que em um mesmo espaço a maior quantidade de um é inversamente proporcional a quantidade do outro e como seus valores de capacidade térmica são muito diferentes (Tabela 1). A maior presença de água corresponde a uma maior capacidade térmica e a maior presença de ar corresponde a uma menor capacidade térmica do solo (HILLEL, 1998).

Tabela 1. Densidade e Capacidade Térmica de constituintes do solo a $10^{\circ} \mathrm{C}$ (Adaptado de HILLEL, 1998).

\begin{tabular}{lcc}
\hline Constituinte & Densidade $(\boldsymbol{\rho})$ & $\begin{array}{c}\text { Capacidade } \\
\text { Térmica }(\boldsymbol{C})\end{array}$ \\
\cline { 2 - 3 } & $\mathbf{( g \cdot \mathbf { c m } ^ { - 3 } )}$ & $\left(\mathbf{c a l} \cdot \mathbf{c m}^{-3} \cdot \boldsymbol{k}\right)$ \\
\hline Quartzo & 2,66 & 0,48 \\
Outros minerais & 2,65 & 0,48 \\
Matéria Orgânica & 1,3 & 0,6 \\
Água (liquida) & 1 & 1 \\
Ar & 0,00125 & 0,003 \\
\hline
\end{tabular}

\subsection{Sensores de condutividade térmica}

Existem diversos tipos de sensores e técnicas desenvolvidas para medir a quantidade de água no solo in situ, a maior parte dessas técnicas consiste na inserção de uma sonda no solo que mede alguma propriedade do solo que é afetada pela presença da água. Exemplos desses sensores são aqueles que medem a constante dielétrica do solo a exemplo do TDR (Time Domain Reflectometry), a sonda de nêutrons, tensiometria, gravimetria, transferência térmica dentre outros (NORIKANE, 2005; BITELLA et al., 2014; FRANCA, 2015).

Os sensores em sua maioria são inviabilizados para uso em campo devido ao elevado custo e também em muitos casos devido à alta complexidade em seu uso, alguns sensores baseados na tensiometria são de manuseio mais simples, porém necessitam de reposição de água constantemente no aparato, assim como a ida frequente no local de instalação para coleta de dados e manutenção, o que limita seu uso (BITELLA et al., 2014; VALENTE et al., 2018). 
Os sensores de fluxo térmico assim como outros que são construídos com componentes eletrônicos são vantajosos por terem um custo mais baixo, menor necessidade de manutenção e menor uso de mão de obra (TARARA et al., 1997; VALENTE et al., 2018; FRANCA, 2015).

O funcionamento dos sensores de fluxo térmico baseia-se na alteração da capacidade térmica do solo devido a um alteração das proporções dos materiais que preenchem os poros, uma vez que a mudança de concentração destes afeta de maneira significativa a capacidade térmica do solo, assim os sensores de capacidade térmica atuam medindo essa variação (HILLEL, 1999; CARSON et al., 2005).

Existem diversos modelos de sensores que utilizam esse princípio na medição da umidade do solo, dentre eles os mais conhecidos são os sensores de ponteira simples (Single Heat Pulse Probe - SPHP) (CAMPBEL, 2006), sensores de ponteiras duplas (Dual Heat Pulse Probe - DHPP) (VALENTE et al., 2004), sensores de ponteiras múltiplas (Multi Heat Pulse Probe - MHPP) (MORI et al., 2003) e sensores tipo botão (Button Heat Pulse Probe - BHPP) (KAMAl et al., 2009).

A construção do sensor é dividida em duas partes principais, um aquecedor e um sensor de temperatura; o aquecedor é responsável por gerar um pulso de calor por um período de tempo fixo e esse calor aquece o solo em sua volta, já o sensor, que está separado a uma distância fixa e conhecida, detecta a variação da temperatura causada pelo pulso térmico para assim determinar a umidade do solo (MORI et al., 2003; VALENTE et al., 2004; CAMPBEL, 2006; KAMAl et al., 2009).

Segundo Shiozawa e Campbell (1990) a condutividade térmica do solo pode ser determinada utilizando a própria sonda de transferência de calor, desde que se saiba qual a quantidade de calor e emitida no pulso térmico, como demonstrado na Equação 1:

$$
\Delta T=T_{f}-T_{0}=-\frac{q}{4 \pi k} \ln \left(t_{f}-t_{0}\right)
$$

Em que $\Delta T\left({ }^{\circ} \mathrm{C}\right)$ a variação de temperatura inicial e final medida no intervalo de tempo $t_{f}-t_{0}(\mathrm{~s}), q$ é a quantidade de calor aplicado por unidade de comprimento da ponteira $\left(W m^{-1}\right), k$ é a condutividade térmica medida $\left(W m^{-1}{ }^{\circ} \mathrm{C}^{-1}\right)$. Assim é possível determinar a condutividade térmica do solo em diferentes condições de umidade. 


\subsection{Sensores de ponteira simples (SPHP)}

Os modelos Single Probe Heat Pulse sensor (SPHP) em tradução livre "sensor de pulso de calor com ponteira simples" são aqueles em que na sua construção a distância entre a fonte de calor e o sensor de temperatura estão muito próximos a sendo possível que ambos fiquem encapsulados em uma ponteira. A empresa Orbital Technologies Corp., Madison, Wis., desenvolveu um sensor do tipo SPHP para fins de monitoramento de umidade do solo em pesquisas espaciais, esta empresa chamou o seu produto de TMAS (Sistema de Aquisição de temperatura e umidade do solo) (Figura 2).

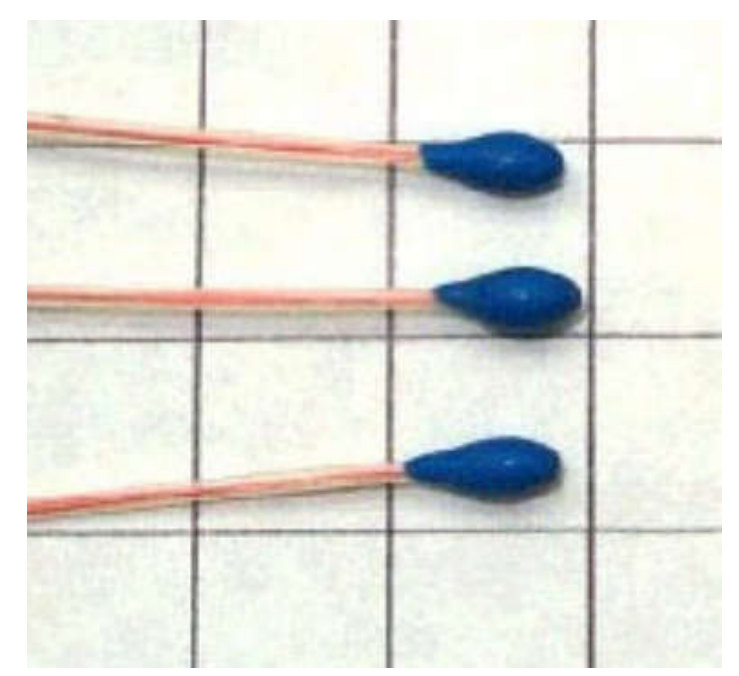

Figura 2. Sensor do tipo TMAS (SPHP) (NORIKANE et al., 2005).

Em estudos feitos por Norikane et al. (2005) os sensores TMAS foram comparados com sensores do tipo TDR ECH2O e Tensiométrico. Os resultados apresentados mostram que o $\mathrm{ECH} 2 \mathrm{O}$ e o Tensiômetro possuem uma faixa ideal de precisão, já o TMAS tende a manter uma leitura constante durante todo processo de secagem do solo, porem tente a superestimar a umidade em alguns pontos.

\subsection{Sensores de ponteira dupla (DHPP)}

Os sensores de ponta dupla são os mais antigos, conhecidos e estudados no que tange os sensores de transferência térmica, a particularidade desses sensores e que são construídos com duas agulhas separadas por uma distância fixa, dentro de uma das agulhas é colocado o elemento aquecedor enquanto na outra e colocado o sensor de temperatura (BRISTOW et al., 1994). 
Os sensores de ponta dupla utilizam Equação 2 proposta por Bristow (1994) em que a variação térmica determina a quantidade de água no solo.

$$
\theta_{V}=\frac{\frac{q^{\prime}}{4 \pi k(\Delta T)}\left[E i\left(\frac{-r^{2}}{4 k\left(t_{m}-t_{0}\right)}\right)-E i\left(\frac{-r^{2}}{4 k\left(t_{m}\right)}\right)\right]-\rho_{b} c_{s}}{\rho_{w} c_{w}}
$$

Onde $\mathrm{k}$ e a difusividade térmica $\left(m^{2} s^{-1}\right), t_{m}$ é o tempo (s) necessário para se atingir a maior temperatura, $\Delta \mathrm{T}\left({ }^{\circ} \mathrm{C}\right)$ é a variação térmica medida no sensor de temperatura, $t_{0}$ é a duração (s) do pulso de calor, $r$ e a distância (m) entre a agulha emissora do pulso térmico e o sensor, q' é a quantidade de calor emitida no pulso térmico por unidade de distância $\left(W m^{-1}\right)$ e Ei $(x)$ integral é a integral exponencial definida pela Equação 3:

$$
E i(x)=-\int_{-x}^{\infty} \frac{e^{-t}}{t} d t
$$

Apesar de eficiente na determinação da umidade do solo, o método da ponteira dupla é de difícil construção, o que inviabiliza sua produção em larga escala (BRISTOW, 1998; BASSINGER et al., 2003; WEN et al., 2015). Valente et al. (2018) propuseram um modelo onde não apenas os as duas agulhas ficam na sonda, mas também todo o circuito necessário para fazer todas as leituras, o que facilitaria a produção do mesmo em larga escala (Figura 3).

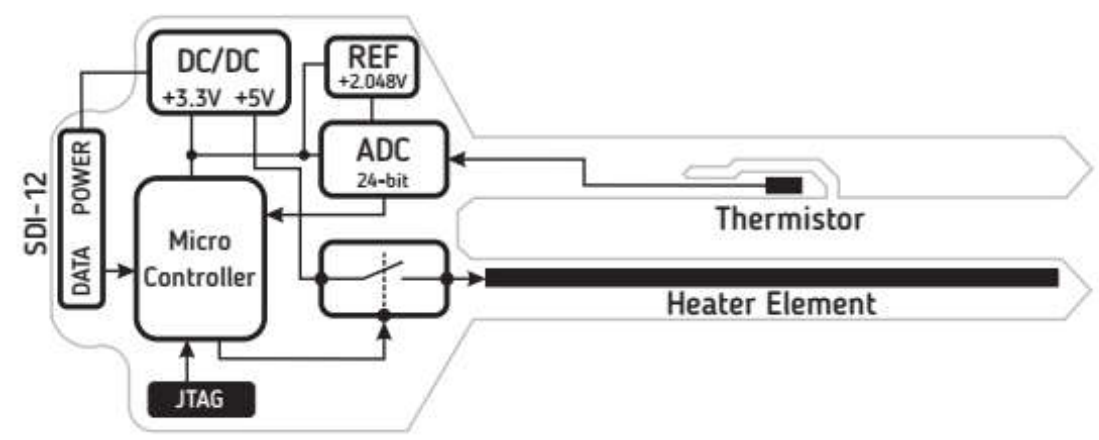

Figura 3. Sensor integrado de umidade do solo onde o circuito de controle fica junto as pontas responsáveis pela medida (VALENTE et al., 2018).

Os resultados obtidos com esse sensor se mostraram promissores, porém o fato de se ter um circuito para cada sensor acaba o deixando mais oneroso, comprometendo assim o princípio de sensor de baixo custo.

Como demonstrado por Palaparthy et al. (2018) os sensores de ponteira dupla, apesar de terem uma boa precisão acabam sendo muito dependentes da forma de 
instalação pois necessitam que as agulhas tenham um bom contato com o solo e também dependem que nenhum grande vazio exista na distância entre as agulhas e para resolver esse tipo de problema outros dois tipos de sensores foram criados.

\subsection{Sensores de múltiplas ponteiras (MHPP) e (BHPP)}

Para solucionar os problemas dos sensores de ponteira dupla alguns pesquisadores criaram os modelos com múltiplas ponteiras onde foram inseridos mais sensores de temperatura de forma que minimizasse o erro que poderia ocorrer da falta de contato com o solo quando havia apenas uma ponteira principal (MORTENSEN et al., 2005) (Figura 4).

A sonda de múltiplas pontas consegue corrigir os problemas da proposta anterior, porem tanto a sua construção quanto sua utilização são bem mais complexas, uma vez que agora existem mais distâncias para serem levadas em consideração e a variação de temperatura é dada por uma média de todas as variações medidas (PALAPARTHY et al., 2018).

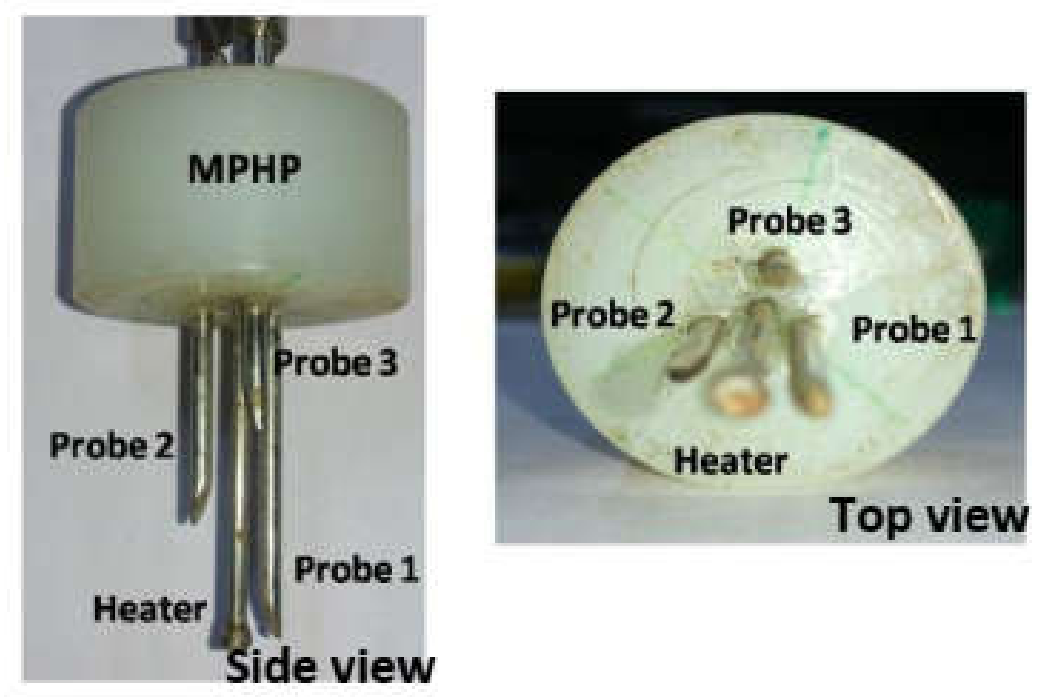

Figura 4. Modelo de MHPP proposto por Palaparthy et al. (2018), utilizando uma ponta aquecedora e três com sensores de temperatura.

Outra forma de tentar minimizar os efeitos dos erros inerentes desse tipo de sensor foi o sensor que utiliza anel metálico oco ao invés de agulhas com elementos aquecedores. Kamai et al. (2009) construiu um modelo que utiliza um anel de aquecimento em volta do sensor de calor, essa forma de construção garante uma 
melhor distribuição do calor e redução dos fatores que podem ocasionar erros (Figura 5).

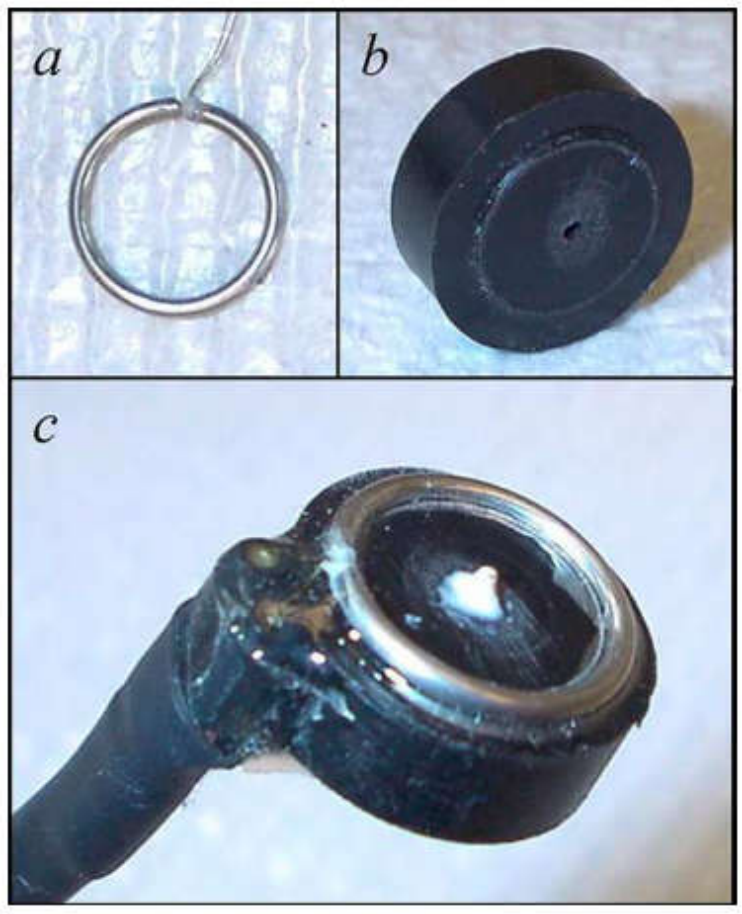

Figura 5. Sensor de temperatura em formato anel: (a) elemento aquecedor, (b) corpo de resina com o orifício central para ser inserido o sensor de temperatura (KAMAl et al., 2009). 


\section{MATERIAIS E MÉTODOS}

\subsection{Local do experimento}

Os experimentos foram realizados na Região Administrativa de Águas Claras entre os meses de abril de maio de 2019.

\subsection{Circuito divisor de tensão}

O sensor construído neste estudo utiliza o princípio de um divisor de tensão onde uma das resistências é variável. O circuito divisor de tensão é formado por duas resistências ligadas em série, e a tensão entre os resistores depende dos valores de cada um, e esta pode ser medida (Figura 6) (SENAI, 1999).

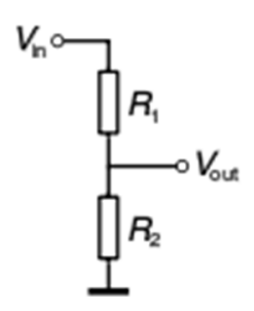

Figura 6. Circuito de divisor de tensão (DIVISOR, 2019).

Como o divisor de tensão foi construído utilizando um resistor de resistência fixa e um de resistência variável (termistor), conforme a resistência do termistor varia o valor da tensão entre os resistores varia e assim é possível determinar o valor da resistência do termistor a uma dada temperatura (ANALOGICA INSTRUMENTAÇÃO E CONTROLE, 2013):

$$
R_{1}=\frac{R_{2} V_{I N}}{V_{O U T}}-R_{2}
$$

Onde $R 1$ e $R 2$ correspondem as resistências dos sensores R1 e R2 respectivamente, Vin é a tensão de entrada e Vout corresponde a tensão na intersecção dos resistores $R 1$ e $R 2$.

A tensão em Volts - que é a tensão lida entre os dois resistores - é obtida pelo conversor ADS1115 (conversor analógico digital) que é capaz de transformar leituras analógicas em leituras digitais utilizando um conversor com resolução de 15 bits. 0 
ADS1115 possui um conversor de 16 bits, porem um dos bits é utilizado para informar se o valor é positivo ou negativo, restando assim 15 bits para a conversão AD. Os limites mínimos e máximos de leitura vão de $\pm 0,256 \mathrm{~V}$ a $\pm 6,144 \mathrm{~V}$ para esse conversor, ou seja ele converte esse intervalo analógico de 0 a $6,144 \mathrm{~V}$ em um intervalo digital que varia de 0 a 32.767, sendo assim cada variação do valor digital corresponde a 0,1875mV como demonstrado na equação abaixo (TEXAS INSTRUMENTS, 2018).

$$
\text { Resolução }=\frac{V_{\text {fundo de escala }}}{2^{N}-1}=\frac{6,144}{2^{15}-1}=\frac{6,144}{32767}=0,1875 \mathrm{mV}
$$

Em posse do valor digital lido pelo conversor AD é possível convertê-lo em $m V$ o multiplicando pela Resolução encontrada na equação 5 e depois encontrando o valor de R1 (Resistência do Termistor) utilizando a equação 4. Com o valor da resistência do termistor naquele momento é possível encontrar o valor da temperatura utilizando a equação de Steinhart-Hart (STEINHART; HART, 1968):

$$
\frac{1}{T}=A+B \ln R+C(\ln R)^{3}
$$

A qual para o sistema em questão pode ser escrita na forma de matriz.

$$
\left[\begin{array}{ccc}
1 & \ln R_{1} & \ln ^{3} R_{1} \\
1 & \ln R_{2} & \ln ^{3} R_{2} \\
1 & \ln R_{3} & \ln ^{3} R_{3}
\end{array}\right]\left[\begin{array}{c}
A \\
B \\
C
\end{array}\right]=\left[\begin{array}{c}
\frac{1}{T_{1}} \\
\frac{1}{T_{2}} \\
\frac{1}{T_{3}}
\end{array}\right]
$$

Em que R1, R2 e R3 são resistências conhecidas nas temperaturas T1, T2 e T3 respectivamente.

Utilizando-se a equação de Steinhart-Hart foi possível obter os seguintes valores de A, B e C para o Termistor utilizado no experimento, os valores de R1, R2 e R3 assim com T1, T2 e T3 foram obtidos no datasheet do termistor.
$A=0,002372645728$
$B=0,00003194067661$
$C=0,0000008795233854$ 


\subsection{Construção do sensor de umidade do solo}

O esquema de construção do sensor é apresentado na Figura 7. Para construir o sensor foi utilizado uma placa universal perfurada de $8 \times 2 \mathrm{~cm}$, a qual foi cortada (Figura 8) e nela foram soldados um termistor SMD TSM10103 (termistor de $10 \mathrm{k} \Omega \mathrm{e}$ 1\%) (THINKING ELECTRONIC INDUSTRIAL CO., LTD., 2018) e um resistor SMD de $10 \mathrm{k} \Omega$ e $1 \%$ com resistência a temperatura formando o circuito divisor de tensão Foram ainda soldados dois resistores de filme metálico de $33 \Omega$ e $2 \mathrm{~W}$ de dissipação em série formando o circuito aquecedor.

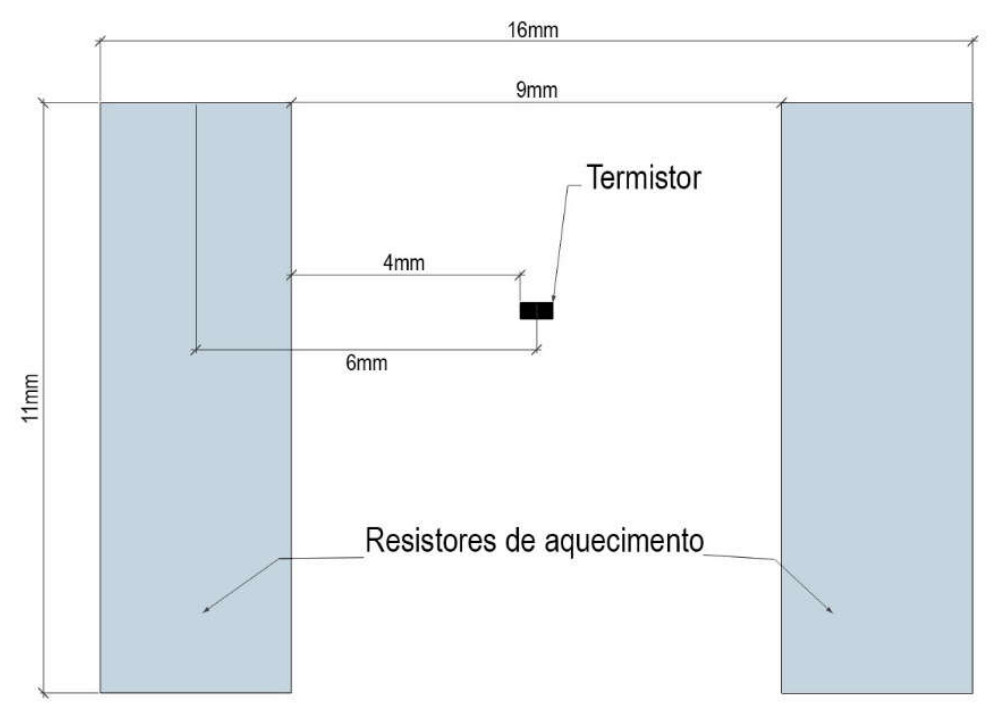

Figura 7. Medidas e posicionamento dos componentes de aquecimento (Resistores de aquecimento) e leitura de temperatura (Termistor).

Para proteger os circuitos o sensor foi encapsulado se utilizando uma resina epóxi SQ2119 com endurecedor SQ3454. O endurecedor utilizado é uma resina comumente utilizada para encapsulamento de eletrônicos visto suas propriedades de isolamento elétrico, alto coeficiente térmico, alta resistência a impactos e intempéries (Figura 9). 


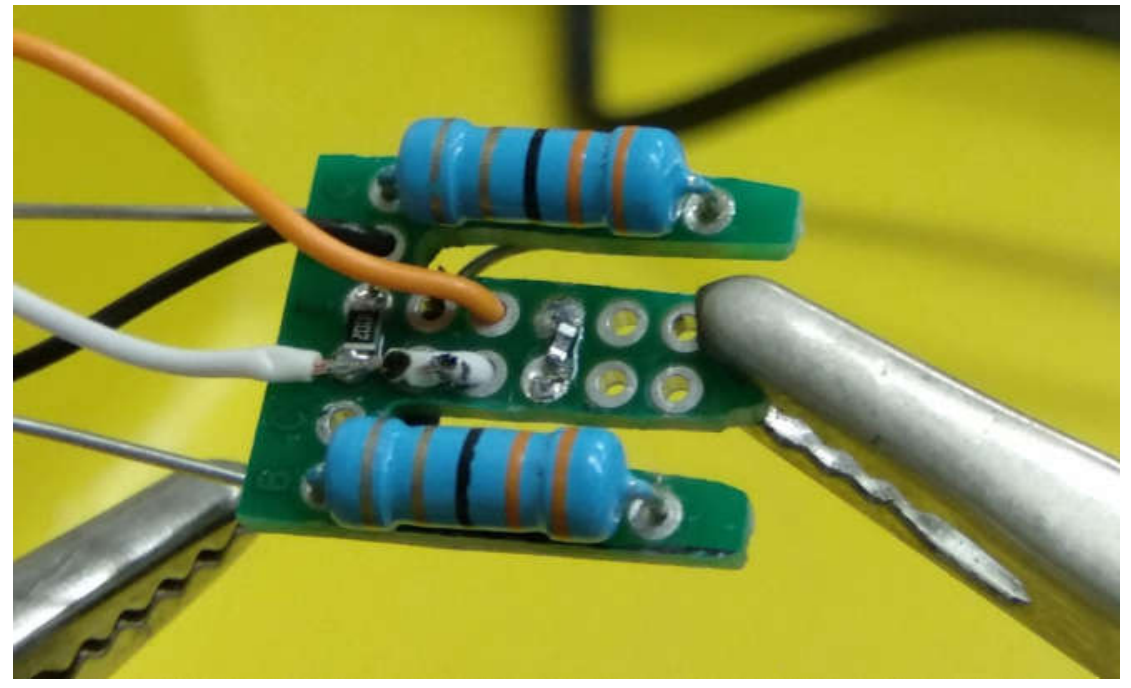

Figura 8. Processo de construção do sensor, soldagem do termistor, resistor de 10k e resistores de aquecimento (azuis).

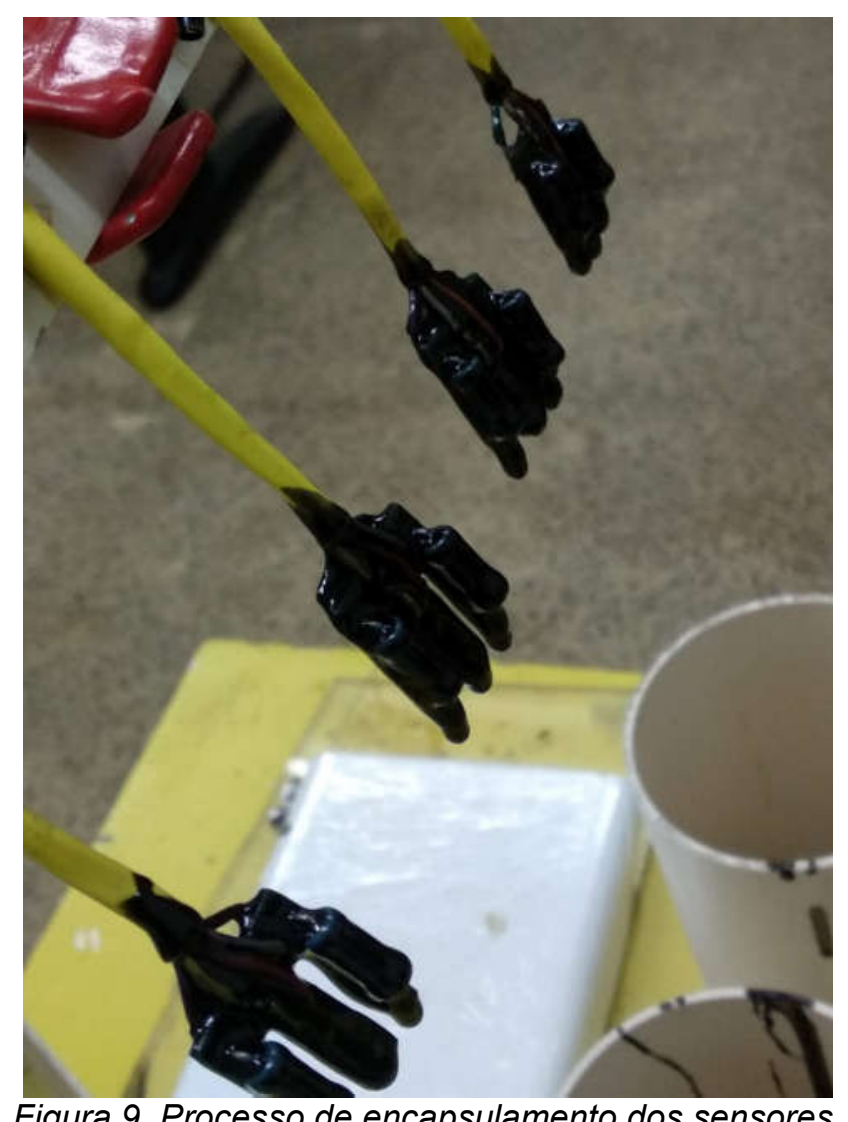

Figura 9. Processo de encapsulamento dos sensores.

O processo de encapsulamento foi feito mergulhando os sensores várias vezes na mistura epóxi, uma vez que por ter uma baixa viscosidade houve uma dificuldade 
em formar uma camada capaz de proteger todas as partes eletrônicas. A placa com os componentes do sensor já montados é apresentada na Figura 10.

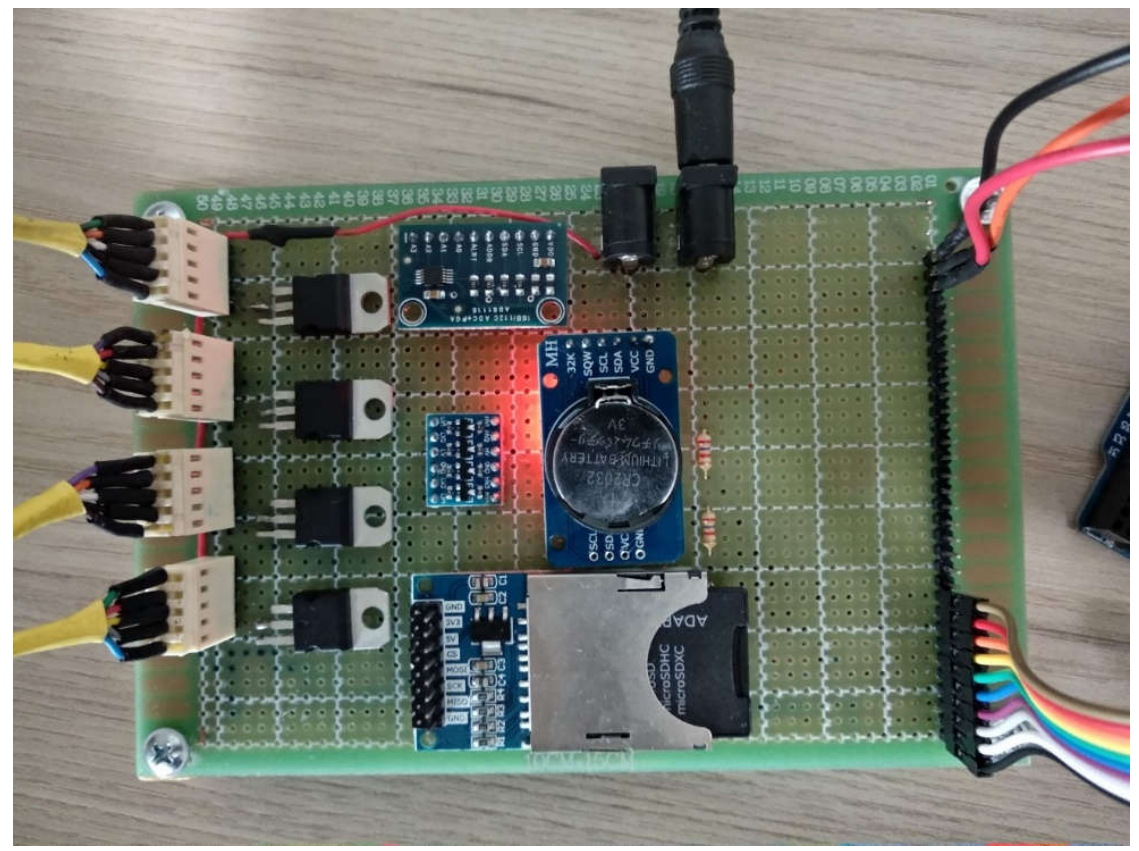

Figura 10. Placa onde foram incorporados todos os componentes necessários para o funcionamento dos sensores.

O circuito de aquecimento formado por 2 resistores iguais ligados em série possuindo cada um $33 \Omega$ com $1 \%$ de tolerância podendo dissipar até $2 \mathrm{~W}$, sendo alimentado por uma fonte de 12 volts e 1,5 amperes.

Lei de ohm (CORRADI, 2010):

$$
R=\frac{V}{I}
$$

Onde:

$R$ é a resistência medida em ohm $(\Omega)$;

l é a intensidade da corrente elétrica, medida em ampere $(A)$;

$\checkmark$ é a diferença de potencial elétrico, medida em volt $(V)$;

Como a resistência e a voltagem são conhecidas, para descobrir o valor da corrente elétrica desse sistema isola-se o $I$.

$$
I=\frac{V}{R}
$$

Desse modo encontra-se o valor de 0,182 A ou $182 \mathrm{~mA}$ (miliampere), valor esse que foi confirmado com o uso de um multímetro modelo UNI-T UT136B. 
A fórmula da potência elétrica é dada por (CORRADI, 2010):

$$
P=R i^{2}
$$

Onde:

$P$ é a potência medida em Watt (W).

Como resultado tem-se uma potência de 1,093 W para cada resistor, como ele possui um limite de $2 \mathrm{~W}$ então o valor dissipado está dentro da faixa de operação do resistor.

\subsection{Funcionamento dos sensores}

Para se testar o funcionamento dos sensores foi utilizada uma amostra de areia que foi peneirada em peneira de $2 \mathrm{~mm}$, seca em estufa de ventilação forçada por 24 horas. Uma amostra de 400 gramas foi saturada com água para determinar a máxima saturação desta quantidade de areia, chegando-se ao valor aproximado de $100 \mathrm{ml}$.

Posteriormente foram separados 4 recipientes com 400 gramas de areia, mediu-se a altura dessa areia e em cada recipiente foi inserido um sensor de forma que o termistor ficasse o mais centralizado possível, tanto em profundidade quanto lateralmente.

Para controlar os sensores foi utilizado uma placa controladora de software e hardware livres, Arduino modelo MEGA 2560 junto ao já citado conversor ADS115, um RTC Real-time Clock (relógio de tempo real), um adaptador para cartão de memória SD para salvar as leituras geradas pelos sensores, também foram utilizados transistores do modelo TIP122 para serem usados como chave de acionamento do pulso térmico.

O programa criado para o Arduino (Anexo 01) foi feito de maneira a executar um processo de aquecimento a cada duas horas, esse prazo foi definido para que houvesse tempo para resfriamento completo do sensor e do solo em volta evitando assim que o calor fosse sendo acumulado de uma leitura para outra.

O experimento foi separado em cinco etapas, em cada etapa foi realizado um conjunto de leituras para determinar se as leituras tinham estabilidade. Na primeira etapa as leituras foram feitas em areia seca e cada uma das seguintes foram acrescentados $20 \mathrm{ml}$ de água, cada leitura é composta por um conjunto de leituras 
anteriores ao pulso de aquecimento, um pulso de aquecimento com tempo definido e posteriormente uma leitura por mais seiscentos segundos que acompanha as alterações da temperatura (Figura 11).

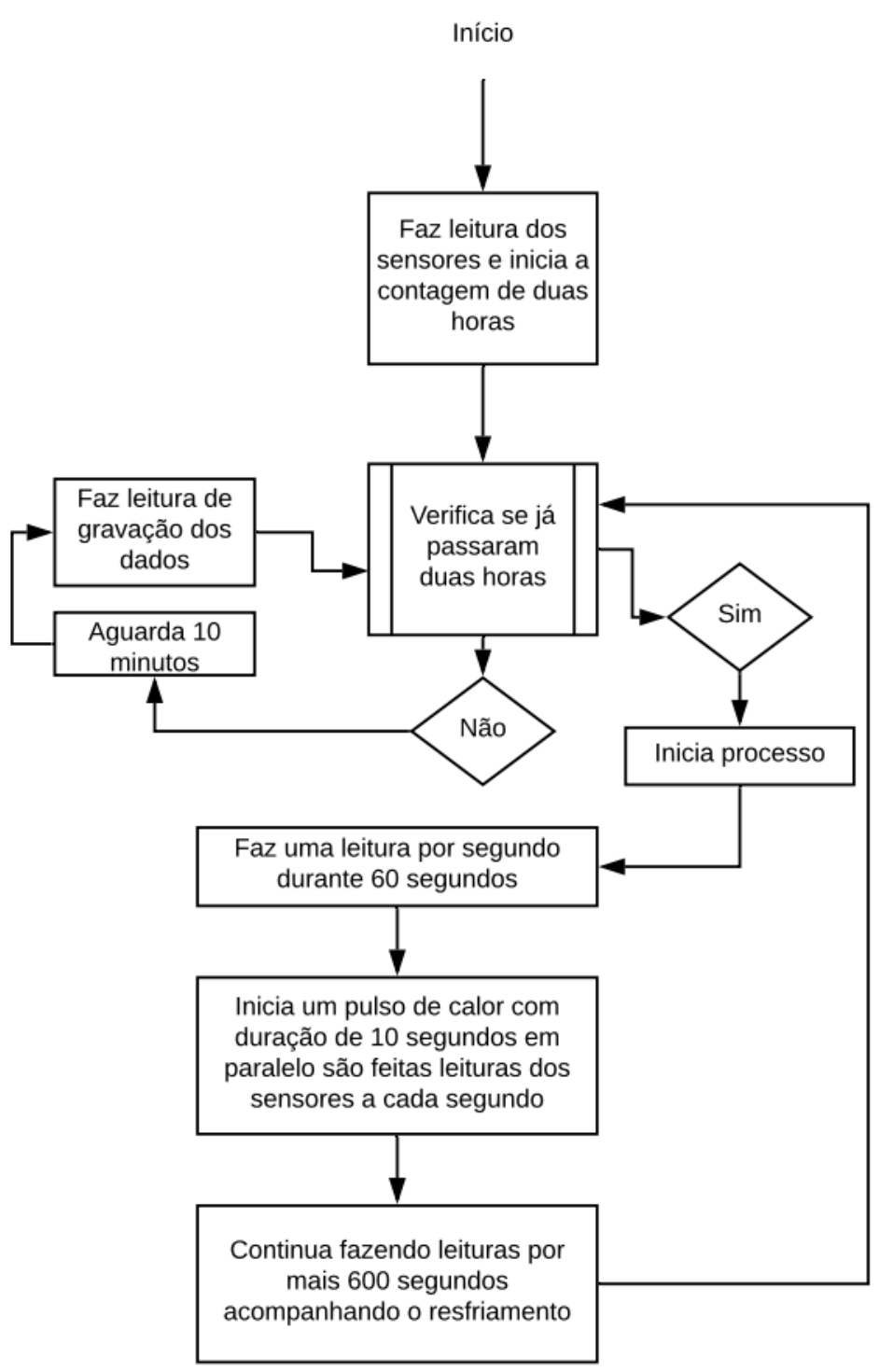

Figura 11. Fluxograma de trabalho do programa que gerencia os sensores. 


\section{RESULTADOS E DISCUSSÃO}

\subsection{Avaliação de precisão e estabilidade de leituras}

Durante o experimento as leituras dos sensores se mostraram muito estáveis assim como o funcionamento geral de todo o sistema, para análise foi utilizada uma média dos primeiros sessenta valores, essa quantidade de leituras foi escolhida para garantir a maior precisão do valor inicial, então o aquecedor era ligado por mais dez segundos e após todo processo decorrido uma análise dos dados foi feita. A resposta de variação de temperatura em função do tempo com a aplicação do pulso de calor para uma das unidades construídas nesse estudo é apresentada na Figura 12.

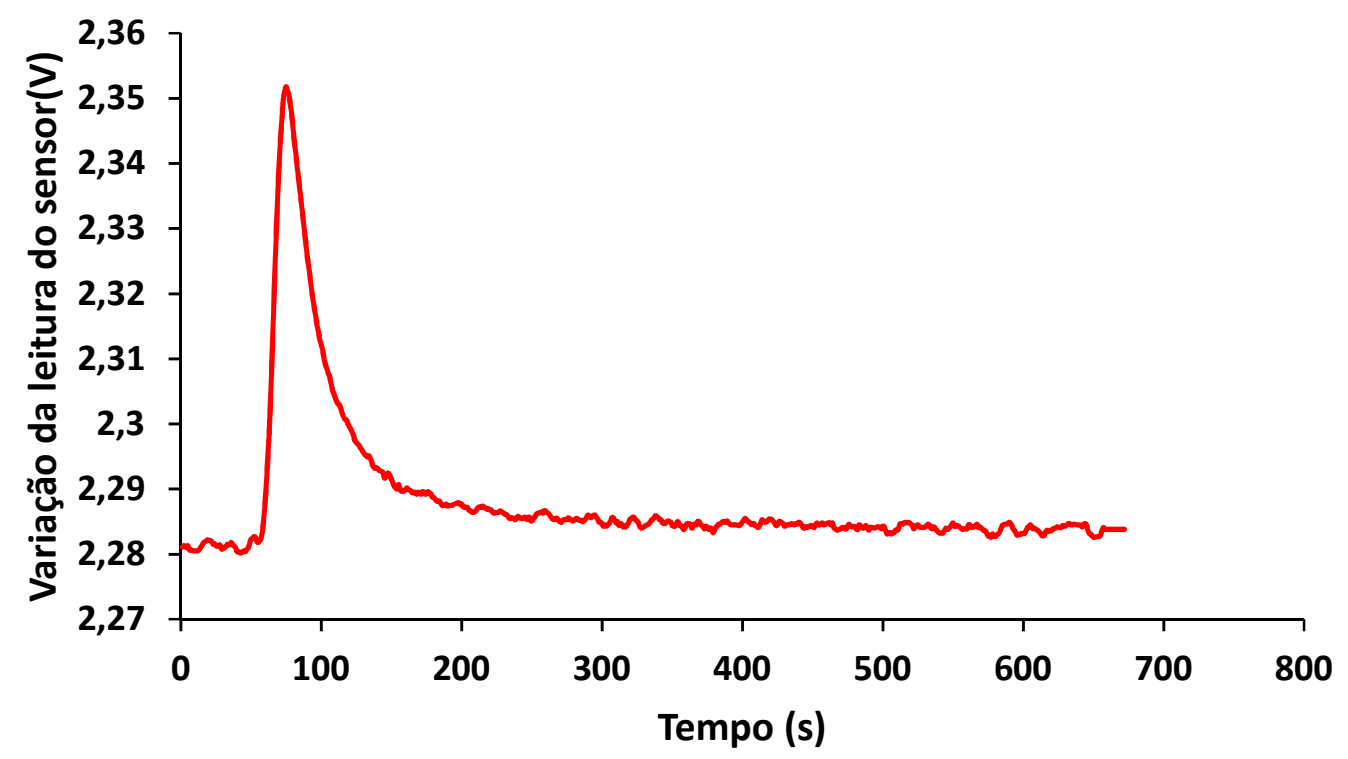

Figura 12. Curva de aquecimento registrada durante o processo de medida da umidade da areia, o resultado analisado consiste na variação em volts entre o pico de calor detectado e a média dos primeiros 60 valores;:

Quanto a variação da temperatura, percebe-se que em situações onde a umidade está acima de $60 \%$ a capacidade do sensor de diferenciar a quantidade de água tende a cair (Figuras 13, 14 e 15), sendo esse um fator que pode limitar a utilização deste sensor.

Essa redução na sensibilidade em alta umidade pode ter sido ocasionada devido a uma baixa emissão de calor, é possível que esse problema seja corrigido com a utilização de um período maior de aquecimento de forma a conseguir uma maior variação entre a temperatura inicial e o pico. 


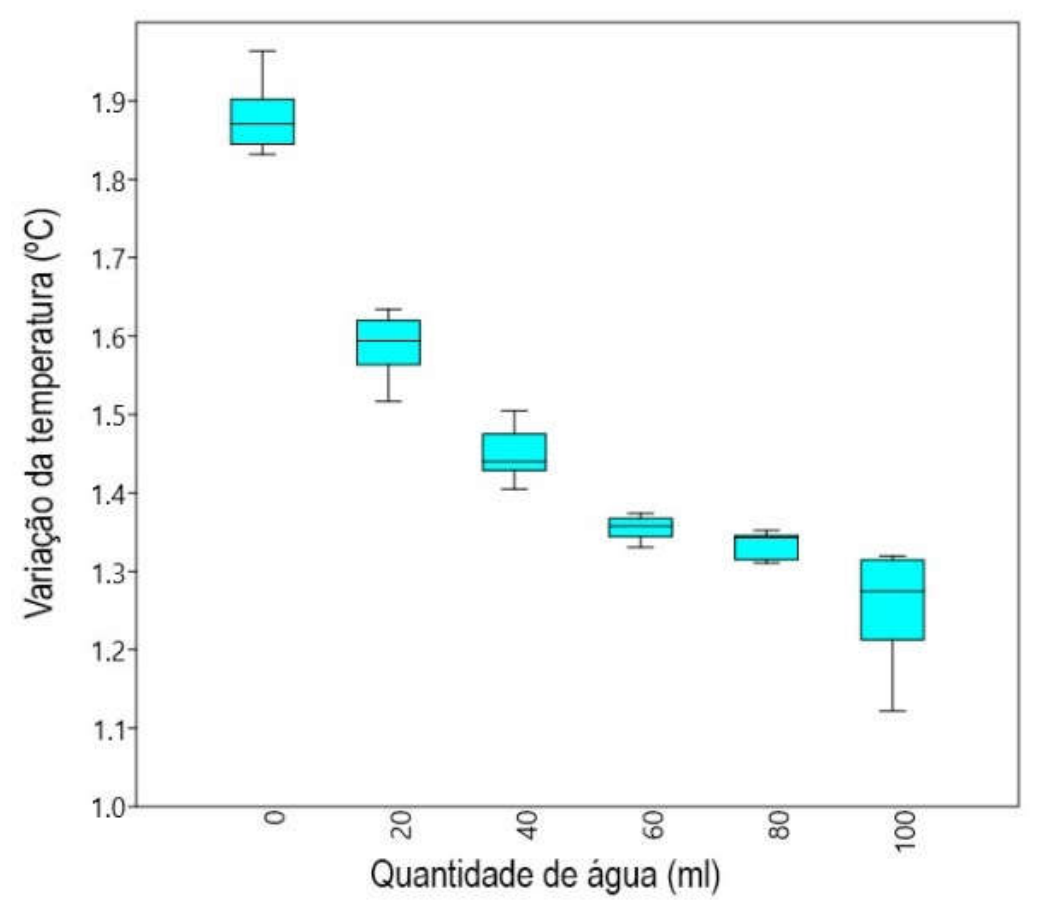

Figura 13. Variação de temperatura em função da quantidade de água aplicada na amostra de areia para o Sensor 01.

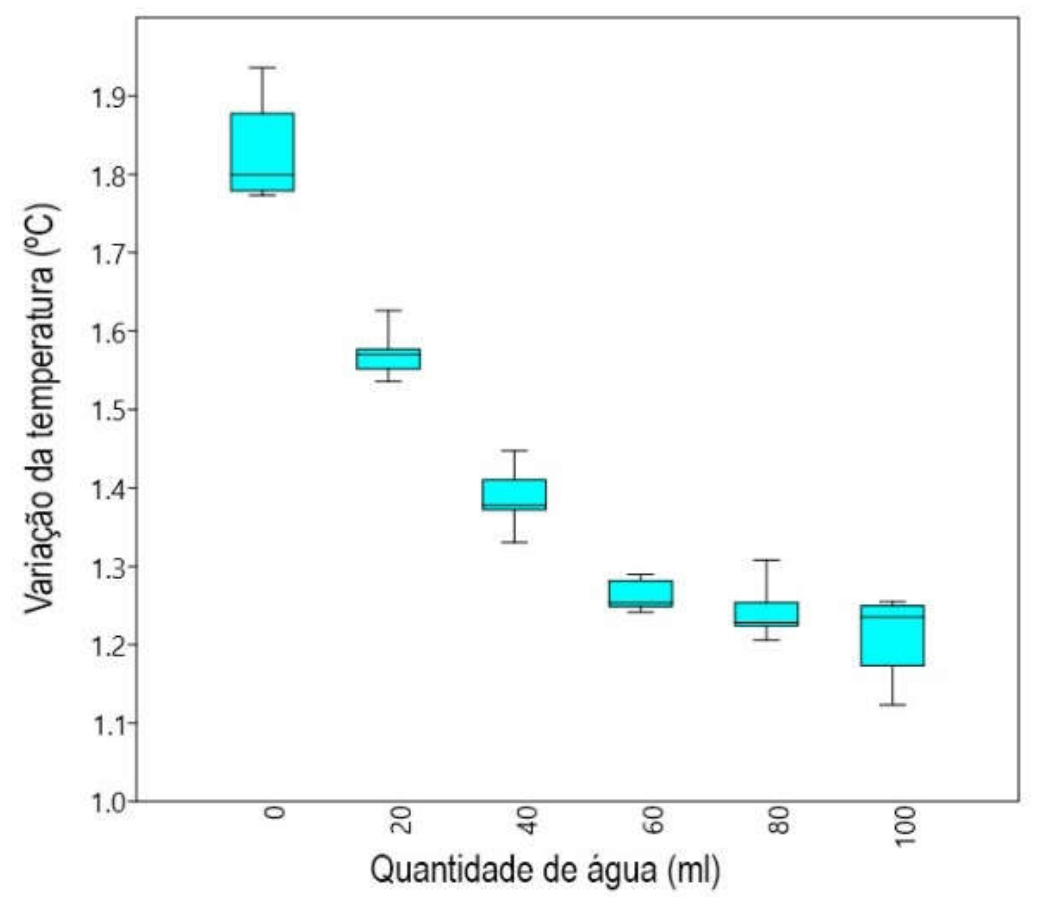

Figura 14. Variação de temperatura em função da quantidade de água aplicada na amostra de areia para o Sensor 02. 


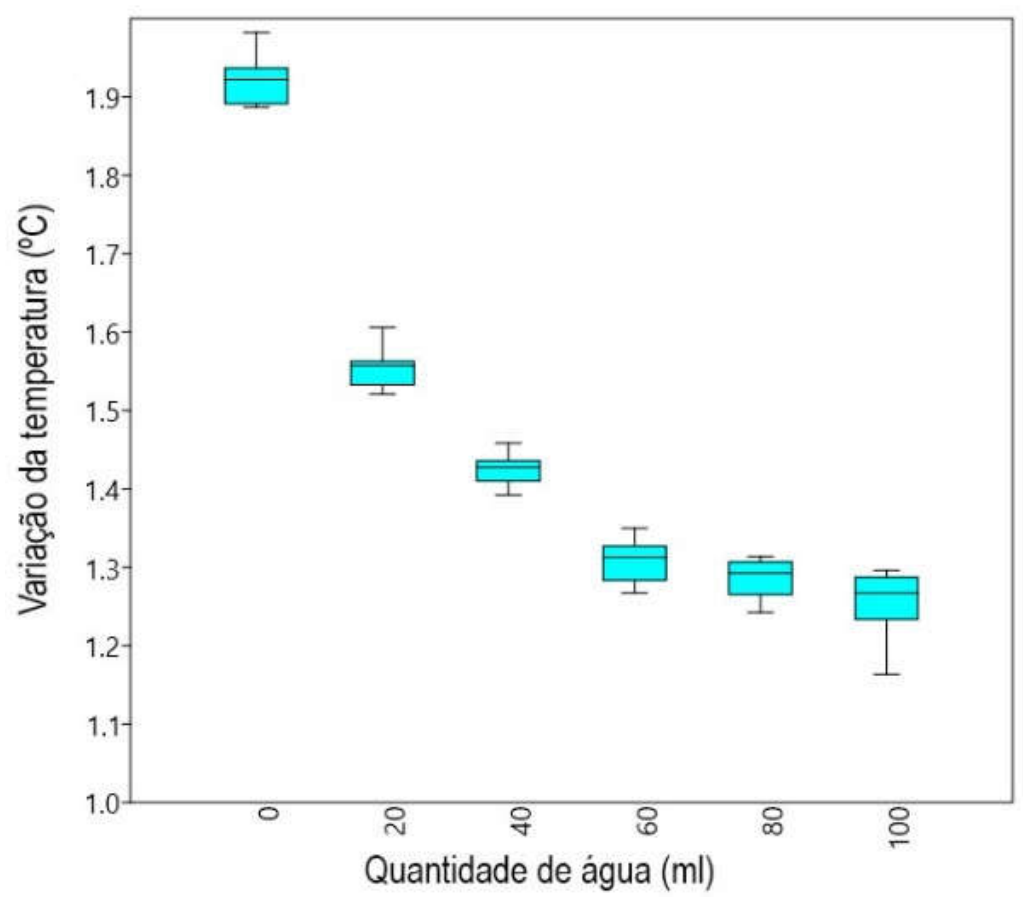

Figura 15. Variação de temperatura em função da quantidade de água aplicada na amostra de areia para o Sensor 03.

$\mathrm{Na}$ literatura a equação proposta por Bristow (1994) (equação 2) é muito utilizada para fins de comparação entre os sensores de uma, duas ou múltiplas pontas, porem verificou-se que essa fórmula não se aplica a este modelo de construção.

Um dos fatores que impedem que esse sensor seja comparado é a dificuldade em se determinar qual é o raio equivalente (distância entre os emissores de calor e sensor). Nas sondas do tipo DHPP, SPHP e MHPP o emissor de calor é uma resistência de Níquel-Cromo, que é um material com diâmetro constante o que facilita o modelamento matemático para uso da equação de Bristow (1994).

Já o sensor proposto neste trabalho, ao utilizar materiais de menor custo e maior facilidade de manejo, utilizou resistores de filme metálico que devido a um formato irregular faz com que a distribuição da temperatura seja também irregular, dificultando a mensuração de um raio equivalente a todos os raios possíveis. 


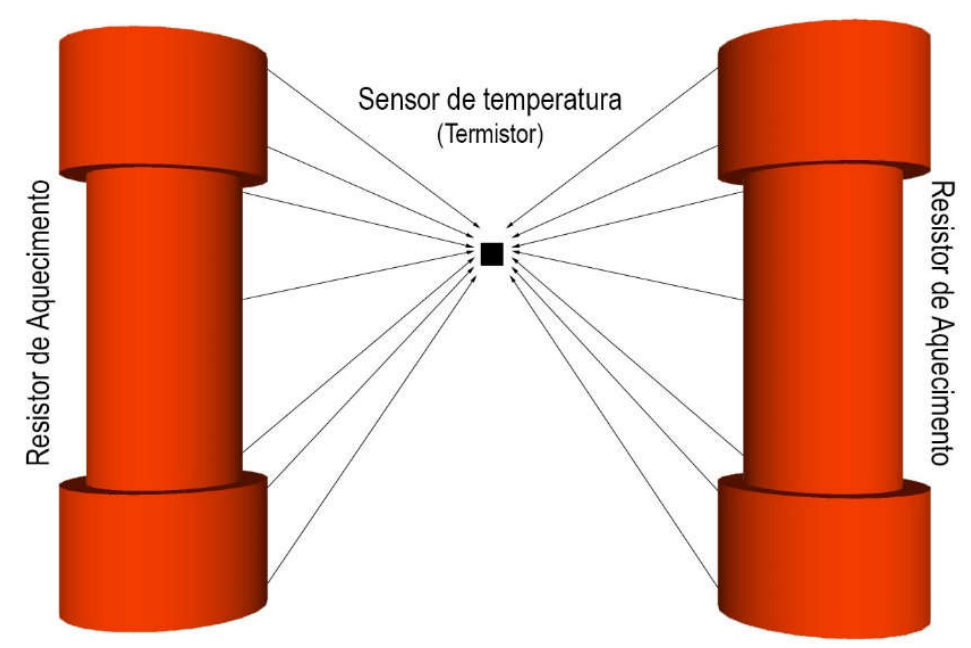

Figura 16. Propagação de calor a partir dos resistores de aquecimento até o termistor.

Alguns pesquisadores tentaram contornar essa limitação ao tentar mensurar empiricamente a quantidade de calor que chega no sensor de temperatura utilizando um meio padrão de ágar (VALENTE et al., 2018). Conhecendo as propriedades térmicas do meio é possível saber a quantidade de calor emitido a partir da variação da temperatura. No entanto esta análise não foi possível para o sensor proposto neste estudo em virtude de problemas operacionais e de cronograma de desenvolvimento.

Kamai et al. (2009) ao propor seu modelo de BHPP utilizou um software para simular a propagação de calor e assim determinar a potência do sistema e posteriormente mensurar a umidade do solo.

Como não foi encontrada na literatura uma forma de padronização deste modelo de sensor de fluxo térmico com outros para se fazer uma comparação, foi utilizada uma equação generalizada de decaimento exponencial para ajuste dos dados na forma:

$$
\Delta T=\Delta T_{\min }+\left(\frac{\Delta T_{\max }-\Delta T_{\min }}{\exp (k \theta)}\right)
$$

Em que: $\theta$ é a umidade volumétrica do solo $\left(\mathrm{m}^{3} \mathrm{~m}^{-3}\right), \Delta T_{\max }$ é a variação de temperatura máxima prevista quando $\theta$ tende a zero e $\Delta T_{\min }$ é a variação de temperatura prevista mínima quando $\theta$ tende a infinito. $O$ ajuste por regressão não linear da Equação 11 aos dados resultou em: 


$$
\Delta T=1,20866+\left(\frac{1,87801-1,20866}{\exp (9,94459 \theta)}\right)
$$

Com todos os coeficientes de ajuste significativos $(p<0.01)$ e $R^{2}=0,86$.

Rearranjando a equação 12, é possível estimar a umidade da variação de temperatura para os sensores desenvolvidos neste estudo utilizando (Figura 16):

$$
\theta=\frac{1}{9,94459} \ln \left(\frac{1,87801-1,20866}{\Delta T-1,20866}\right)
$$

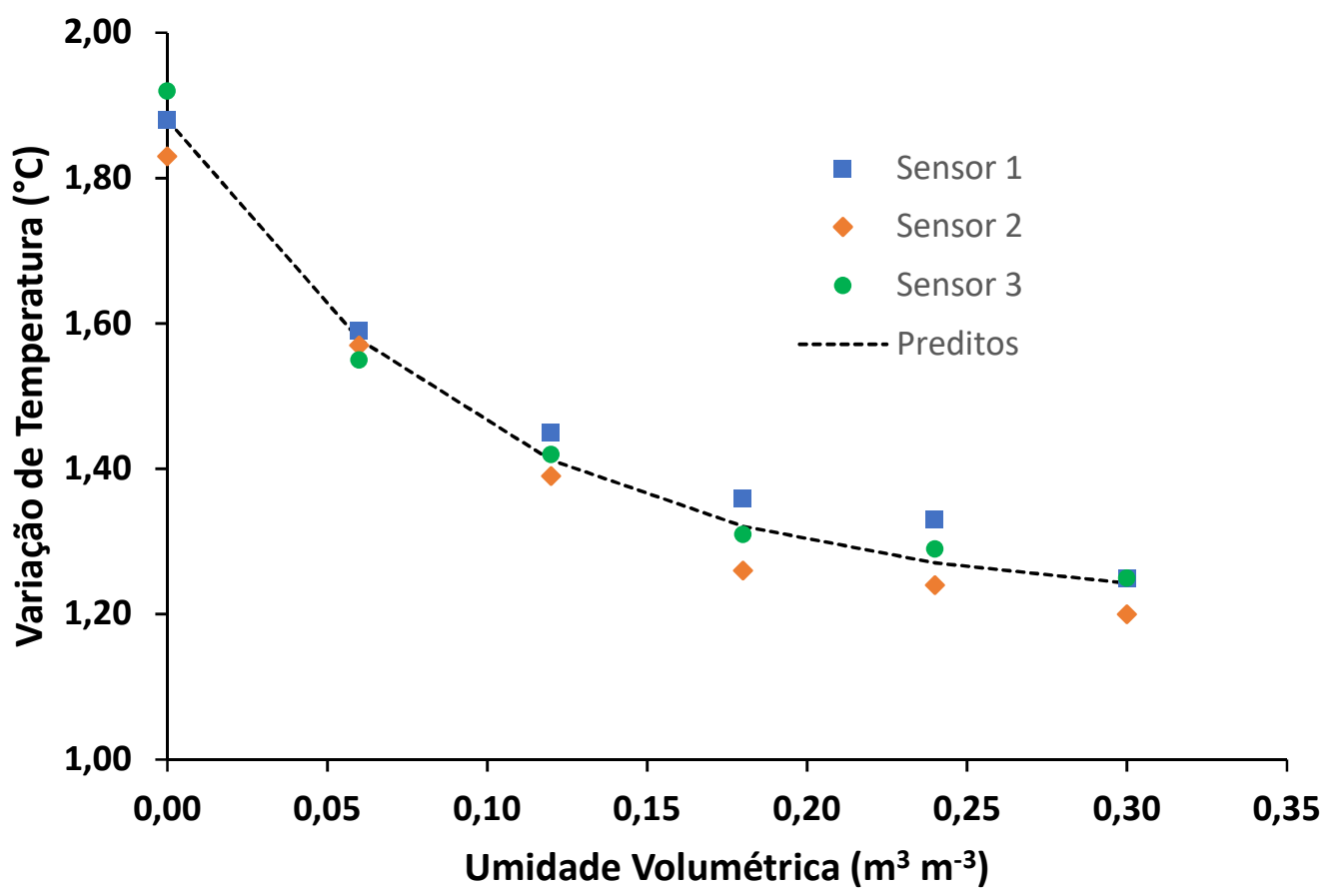

Figura 17. Variação de temperatura previstos pela equação (12) e valores medidos pelos sensores em função da umidade volumétrica do solo

\subsection{Análise de custo}

Uma das vantagens desse sensor e que todos os componentes podem ser encontrados com facilidade no Brasil, tendo também uma variedade de fornecedores já que em sua maioria são hardwares livres (Tabelas 2 e 3). 
Tabela 2. Custos aproximados dos componentes da controladora das sondas.

\begin{tabular}{|c|c|c|c|c|c|}
\hline ITEM & Unidade & Quantidade & Preço & \multicolumn{2}{|c|}{ Total } \\
\hline ARDUINO UNO & Und. & 1 & $\begin{array}{l}\mathrm{R} \$ \\
30,00\end{array}$ & \multicolumn{2}{|c|}{$\begin{array}{l}\mathrm{R} \$ \\
30,00\end{array}$} \\
\hline RTC DS3231 & Und. & 1 & $\begin{array}{l}\mathrm{R} \$ \\
13,00\end{array}$ & \multicolumn{2}{|l|}{$\begin{array}{l}\mathrm{R} \$ \\
13,00\end{array}$} \\
\hline SHIELD DE CARTÃO SD & Und. & 1 & $\begin{array}{l}\mathrm{R} \$ \\
8,00\end{array}$ & $\mathrm{R} \$$ & 8,00 \\
\hline CARTÃO SD 8 GB & Und. & 1 & $\begin{array}{l}R \$ \\
20,00\end{array}$ & \multicolumn{2}{|l|}{$\begin{array}{l}\mathrm{R} \$ \\
20,00\end{array}$} \\
\hline ADS1115 & Und. & 1 & $\begin{array}{l}\mathrm{R} \$ \\
25,00\end{array}$ & \multicolumn{2}{|l|}{$\begin{array}{l}\mathrm{R} \$ \\
25,00\end{array}$} \\
\hline TRANSISTOR TIP 120 & Und. & 4 & $\begin{array}{l}\mathrm{R} \$ \\
1,22\end{array}$ & $\mathrm{R} \$$ & 4,88 \\
\hline CONECTORES & Und. & 4 & $\begin{array}{l}\mathrm{R} \$ \\
1,50\end{array}$ & $\mathrm{R} \$$ & 6,00 \\
\hline FIAÇÃO & Metro & 1 & $\begin{array}{l}\mathrm{R} \$ \\
2,50\end{array}$ & $\mathrm{R} \$$ & 2,50 \\
\hline $\begin{array}{l}\text { PLACA UNIVERSAL } \\
\text { PERFURADA 10X15CM }\end{array}$ & Und. & 1 & $\begin{array}{l}\mathrm{R} \$ \\
15,00\end{array}$ & \multicolumn{2}{|l|}{$\begin{array}{l}\mathrm{R} \$ \\
15,00\end{array}$} \\
\hline $\begin{array}{l}\text { CONVERSOR DE NÍVEL } \\
\text { LÓGICO 5V PARA 3,3V }\end{array}$ & Und. & 1 & $\begin{array}{l}\mathrm{R} \$ \\
5,00\end{array}$ & $\mathrm{R} \$$ & 5,00 \\
\hline RESISTORES 2,2KR & Und. & 4 & $\begin{array}{l}\mathrm{R} \$ \\
0,20\end{array}$ & $\mathrm{R} \$$ & 0,80 \\
\hline FONTE $12 \mathrm{~V} 3 \mathrm{~A}$ & Und. & 1 & $\begin{array}{l}\mathrm{R} \$ \\
25,00\end{array}$ & \multicolumn{2}{|l|}{$\begin{array}{l}\mathrm{R} \$ \\
20,00\end{array}$} \\
\hline \multicolumn{3}{|c|}{ TOTAL } & & \multicolumn{2}{|c|}{$\mathrm{R} \$ \quad 155,18$} \\
\hline
\end{tabular}

Tabela 3. Custos dos componentes necessários para construção de uma unidade da sonda térmica.

\begin{tabular}{|c|c|c|c|c|}
\hline ITEM & Unidade & Quantidade & Preço & Total \\
\hline $\begin{array}{l}\text { RESISTOR 47R 2W } \\
5 \%\end{array}$ & Und. & 2 & $\begin{array}{l}\mathrm{R} \$ \\
0,30\end{array}$ & $\begin{array}{l}\mathrm{R} \$ \\
0,60\end{array}$ \\
\hline $\begin{array}{l}\text { RESISTOR SMD 10KR } \\
1 \%\end{array}$ & Und. & 1 & $\begin{array}{l}\mathrm{R} \$ \\
0,05\end{array}$ & $\begin{array}{l}\mathrm{R} \$ \\
0,05\end{array}$ \\
\hline $\begin{array}{l}\text { TERMISTOR SMD } \\
\text { 10KR } 1 \%\end{array}$ & Und. & 1 & $\begin{array}{l}\mathrm{R} \$ \\
0,30\end{array}$ & $\begin{array}{l}\mathrm{R} \$ \\
0,30\end{array}$ \\
\hline $\begin{array}{l}\text { PLACA UNIVERSAL } \\
2 \times 8\end{array}$ & Und. & 0,3 & $\begin{array}{l}\mathrm{RS} \\
2,50\end{array}$ & $\begin{array}{l}\mathrm{R} \$ \\
0,75\end{array}$ \\
\hline $\begin{array}{l}\text { CABO MANGA } 6 \\
\text { VIAS }\end{array}$ & Metro & 1 & $\begin{array}{l}\mathrm{RS} \\
2,60\end{array}$ & $\begin{array}{l}\mathrm{R} \$ \\
2,60\end{array}$ \\
\hline $\begin{array}{l}\text { RESINA EPOXY } \\
\text { SQ2119 + SQ5134 }\end{array}$ & Gramas & 2,5 & $\begin{array}{l}\mathrm{R} \$ \\
0,10\end{array}$ & $\begin{array}{l}\mathrm{R} \$ \\
0,25\end{array}$ \\
\hline \multicolumn{4}{|c|}{ TOTAL } & $\begin{array}{l}\mathrm{R} \$ \\
4,55\end{array}$ \\
\hline
\end{tabular}

Os preços apresentados nas tabelas 2 e 3 foram cotados em lojas online e físicas em Brasília no ano de 2018 e 2019, não havendo mudanças significativas de 
preços nessas datas. Na tabela 4 apresenta-se os valores cotados nas principais lojas onde os produtos foram adquiridos.

Para construção da sonda foi levado em consideração que com cada placa universal de $2 \times 8 \mathrm{~cm}$ é possível construir 3 sondas, a mesma lógica foi aplicada a resina epóxi que é vendida apenas por $\mathrm{kg}$, mas são necessários aproximadamente 2,5 gramas para cobrir cada sonda, já levando em consideração as perdas.

Cada conversor AD ADS1115 suporta até quatro sondas e cada Arduino suporta até quatro desses sensores, ou seja, é possível ampliar para 12 sondas utilizando a mesma placa de Arduino.

Tabela 4. Valores dos componentes necessários cotados em lojas física e online em junho de 2019.

\begin{tabular}{|c|c|c|c|c|c|c|c|c|}
\hline \multirow{2}{*}{$\begin{array}{l}\text { ITEM } \\
\text { ARDUINO UNO }\end{array}$} & \multicolumn{2}{|c|}{ Mercado Livre } & \multicolumn{2}{|c|}{ Hu Infinito } & \multicolumn{2}{|c|}{$\begin{array}{l}\text { Casa do } \\
\text { resistor }\end{array}$} & \multicolumn{2}{|c|}{ Curto circuito } \\
\hline & $\mathrm{R} \$$ & 29,89 & $\mathrm{R} \$$ & 52,90 & $\mathrm{R} \$$ & 42,76 & $\mathrm{R} \$$ & 27,40 \\
\hline SHIELD DE CARTÃO SD & $\mathrm{R} \$$ & 13,07 & & - & $\mathrm{R} \$$ & 10,44 & $\mathrm{R} \$$ & 4,55 \\
\hline CARTÃO SD 8 GB & $R \$$ & 30,98 & & - & & - & & - \\
\hline ADS1115 & $\mathrm{R} \$$ & 26,32 & & - & & - & $\mathrm{R} \$$ & 21,90 \\
\hline TRANSISTOR TIP 120 & $\mathrm{R} \$$ & 1,85 & $\mathrm{R} \$$ & 1,56 & $\mathrm{R} \$$ & 1,94 & $\mathrm{R} \$$ & 2,15 \\
\hline CONECTORES & $\mathrm{R} \$$ & 2,00 & $\mathrm{R} \$$ & 1,60 & & - & & - \\
\hline FIAÇÃO & $\mathrm{R} \$$ & 3,20 & $\mathrm{R} \$$ & 2,54 & $\mathrm{R} \$$ & 2,74 & & - \\
\hline $\begin{array}{l}\text { PLACA UNIVERSAL } \\
\text { PERFURADA } 10 \times 15 \mathrm{CM}\end{array}$ & $\mathrm{R} \$$ & 19,99 & $\mathrm{R} \$$ & 13,37 & $\mathrm{R} \$$ & 15,00 & $\mathrm{R} \$$ & 8,10 \\
\hline $\begin{array}{l}\text { CONVERSOR DE NÍVEL } \\
\text { LÓGICO 5V PARA 3,3V }\end{array}$ & $\mathrm{R} \$$ & 8,00 & $\mathrm{R} \$$ & 2,90 & & - & $\mathrm{R} \$$ & 3,10 \\
\hline RESISTORES 2,2KR & $\mathrm{R} \$$ & 0,02 & $\mathrm{R} \$$ & 0,04 & $\mathrm{R} \$$ & 0,08 & $\mathrm{R} \$$ & 0,10 \\
\hline FONTE 12V 3A & $\mathrm{R} \$$ & 15,00 & $\mathrm{R} \$$ & 18,90 & $\mathrm{R} \$$ & 28,80 & $\mathrm{R} \$$ & 25,00 \\
\hline RESISTOR 47R 2W 5\% & $\mathrm{R} \$$ & 0,27 & $\mathrm{R} \$$ & 0,39 & $\mathrm{R} \$$ & 0,30 & $\mathrm{R} \$$ & 1,35 \\
\hline RESISTOR SMD 10KR 1\% & $\mathrm{R} \$$ & 0,16 & & - & $\mathrm{R} \$$ & 0,07 & & - \\
\hline $\begin{array}{l}\text { TERMISTOR SMD 10KR } \\
1 \%\end{array}$ & $\mathrm{R} \$$ & 0,30 & & - & & - & & - \\
\hline PLACA UNIVERSAL 2X8 & $\mathrm{R} \$$ & 8,00 & $\mathrm{R} \$$ & 2,90 & $\mathrm{R} \$$ & 3,48 & $\mathrm{R} \$$ & 1,90 \\
\hline CABO MANGA 6 VIAS & $\mathrm{R} \$$ & 3,20 & $\mathrm{R} \$$ & 2,54 & $\mathrm{R} \$$ & 2,74 & & - \\
\hline $\begin{array}{l}\text { RESINA EPOXY SQ2119 + } \\
\text { SQ5134 }\end{array}$ & $\mathrm{R} \$$ & 75,00 & & - & & - & & - \\
\hline
\end{tabular}




\section{CONCLUSÃO}

Os sensores apresentados apresentam uma boa relação de custo-benefício embora ainda não possa diferir o teor de umidade em umidades mais elevadas, o que limita seu uso.

O processo de fabricação se mostrou eficiente, ainda que exista muito trabalho manual, o método não requer equipamentos de precisão para que ocorra, uma vez que as distâncias são baseadas na perfuração da placa.

Ainda que os resultados tenham sido satisfatórios são necessários mais estudos com objetivo de aprimorar a leitura em todos os níveis de umidade assim como a determinação do raio efetivo para que se possa utilizar a equação 2 , isso facilitaria o processo de calibração para outros tipos de solo. 


\section{REFERÊNCIAS}

ALLEN, R. G.; PEREIRA, L. S.; RAES, Dirk.; SMITH, Martin. Crop evapotranspiration - Guidelines for computing crop water requirements. FAO - Food and Agriculture Organization of the United Nations, Roma, p. 1-15, 1998.

ANALOGICA INSTRUMENTAÇÃO E CONTROLE. NOTA TÉCNICA NT-011. BRASIL. 2013.

BASINGER, J. M.; KLUITENBERG, G. J.; FRANK, J. M.; BARNES, P. L.; KIRKHAM, M. B. Laboratory Evaluation of the Dual-Probe Heat-Pulse Method for Measuring Soil Water Content. Vadose Zone Journal, Madison, p. 389-399, 2003.

BITELLA, G.; ROSSI, R.; BOCHICCHIO, R.; PERNIOLA, M.; AMATO, M. A Novel LowCost Open-Hardware Platform for Monitoring Soil Water Content and Multiple Soil-AirVegetation Parameters. sensors, p. 19639 - 19659, 2014.

BRASIL. Ministério da Agricultura, Pecuária e Abastecimento. Portaria $n^{\circ} 852$ - Art. $1^{\circ}$ Criar a Comissão Brasileira de Agricultura de Precisão - CBAP. Diário Oficial da República Federativa do Brasil, Brasília, DF, 21 set. 2012. Seção 1, n. 184.

BRISTOW, K. L. Measurement of thermal properties and water content of unsaturated sandy soil using dual-probe heat-pulse probes. Agricultural and Forest Meteorology, v. 89, p. 75-84, 1998.

BRISTOW, K. L.; KLUITENBERG, G. J.; GODING, C.J.; FITZGERALD, T. S. A small multi-needle probe for measuring soil thermal properties, water content and electrical conductivity. Computers and Electronics in Agriculture, v. 31, p. 265-280, 2001.

BRISTOW, K. L.; KLUITENBERG, G. J.; HORTON, R. Measurement of Soil Thermal Properties with a Dual-Probe Heat-Pulse Technique. Soil Science Society of America, v. 58, p. 1288-1294, 1994.

CAMPBELL SCIENTIFIC, INC. 229 Heat Dissipation Matric Water Potential Sensor. Logan, p. 1-34. 2009.

CARSON, J. K.; LOVATT, S. J.; TANNER, D. J.; CLELAND, A. C. Thermal conductivity bounds for isotropic, porous materials. International Journal of Heat and Mass Transfer, v. 48, p. 2150-2158, 2005.

CENGEL, A. Heat Transfer A Practical Approach. 2. ed. [s.n.], 2007. 334-350 p.

DIAS, P. C.; ROQUE, W.; FERREIRA, E. C.; DIAS, J. A. A high sensitivity single-probe heat pulse soil moisture sensor based on a single npn junction transistor. Computers and Electronics in Agriculture, Campinas, v. 96, p. 138-147, 2013.

DIVISOR DE TENSÃO. In: WIKPÉDIA: a enciclopédia livre. Wikmedia, 2019. Disponivel em: <https://pt.wikipedia.org/wiki/Divisor_de_tens\%C3\%A3o>. Acesso em: 20 mar. 2019. 
EMBRAPA. Embrapa. $\mathbf{O}$ desafio do uso da água na agricultura brasileira. Disponível em: <https://www.embrapa.br/agua-na-agricultura/sobre-o-tema >. Acesso em: 20 mar. 2019.

FRANÇA, Maria Bernadete de Morais. Contribuição ao estudo e fabricação de sensores de umidade do solo usando a técnica da transferência de calor. 2015. $61 \mathrm{f}$. Tese (Doutorado em Engenharia Elétrica) - Faculdade de Engenharia Elétrica e de Computação, Universidade Estadual de Campinas, Campinas, 2015.

GREACEN, E. L. Soil water assessment by the neutron method. Melbourne: Commonwealth Scientific and Industrial Research Organization, Glen Osmond (Australia). Div. of Soils, 1981.

HARGREAVES, G. H.; SAMANI, Z. A. Reference crop evapotranspiration from temperature. Applied engineering in agriculture, v. 1, p. 96-99, 1985.

HILLEL, D. Environmental Soil Physics. San Diego: Academic Press, 1998.

KAMAI, T.; KLUITENBERG, G. J.; HOPMANS, J. W. Design and numerical analysis of a button heat probe for soil water content measurement. Vadose Zone Journal, v. 8 , p. 167-173, 2009.

KITTEL, C.; KROEMER, H. Thermal physics. 2. ed. [S.I.]: [s.n.], 2000. 87-90 p.

LOEB, A. L. Thermal Conductivity: VIII, A Theory of Thermal Conductivity of Porous Materials. Journal of the American Ceramic Society, v. 37, p. 96-99, 1954.

MACHADO, J.; PADILHA, M. R. F.; LIRA, F. P.; OLIVEIRA, J. G.; SILVA, R. S.; CAETANO, M. B. C. Agricultura de Precisão e abertura de novas fronteiras no Brasil. Geama Scientific Journal of Environmental Sciences, Recife, v. 1, n. 4, p. 49-53, Jan-Mar 2018.

MOLIN, J. P. AGRICULTURA DE PRECISÃO: NÚMEROS DO MERCADO BRASILEIRO. Laboratório de Agricultura de Precisão. São Paulo, p. 1-7. 2017.

MORTENSEN, A. P.; HOPMANS, J. W.; MORI, Y.; SIMUNEK, J. Multi-functional heat pulse probe measurements of coupled vadose zone flow and transport. Water Resources, v. 29, p. 250-267, 2006.

NORIKANE, J. H.; PRENGER, J. J.; ROUZAN-WHEELDON, D. T.; LEVINE, H. G. A COMPARISON OF SOIL MOISTURE SENSORS FOR SPACE FLIGHT APPLICATIONS. American Society of Agricultural Engineers, Lexington, v. 21, p. 211-216, 2005.

PALAPARTHY, V. S.; MONDAL, S.; SINGH, D. N.; BAGHINI, M. S.; ANANTHASURESH, G. K. Effect of spatial variations and desiccation cracks on the DPHP and MPHP sensors. Sensors and Actuators A, 2018.

SENAI; CST. Eletrotécnica Básica - Instrumentação. Espírito Santo, p. 2-10. 1999.

SHIOZAWA, S.; CAMPBELL, G. S. Soil thermal conductivity. Remote Sensing Reviews, v. 5, p. 301-310, 1990. 
STEINHART, J. S.; HART, S. R. Calibration curves for thermistors. Deep Sea Research and Oceanographic Abstracts, Elsevier, p. 497-503, 1968.

TARARA, J. M.; HAM, J. M. Measuring Soil Water Content in the Laboratory and Field with Dual-Probe Heat-Capacity Sensors. Agronomy journal, v. 89, p. 535-542, July 1997. ISSN 4.

TEXAS INSTRUMENTS. ADS111x Ultra-Small, Low-Power, I2C-Compatible, 860SPS, 16-Bit ADCs With Internal Reference, Oscillator, and Programmable Comparator. Texas, p. 1-54. 2018.

THINKING ELECTRONIC INDUSTRIAL CO., LTD. NTC Termistor: TSM Series. Taiwan. 2018.

VALENTE, A.; SARAIVA, A. A.; FERREIRA, N. M. F.; SOARES, S. On the Design and Construction of Dual-Probe Heat-Pulse Soil Moisture Sensor: Towards an Industrial Solution. ALLSENSORS 2018: The Third International Conference on Advances in Sensors, Actuators, Metering and Sensing, p. 43 - 48, 2018.

WEN, M.; LIU, G.; LI, B.; SI, B. C.; HORTON, R. Evaluation of a self-correcting dual probe heat pulse sensor. Agricultural and Forest Meteorology, v. 200, p. 203-208, 2015. 


\section{ANEXO}

\section{Anexo 01}

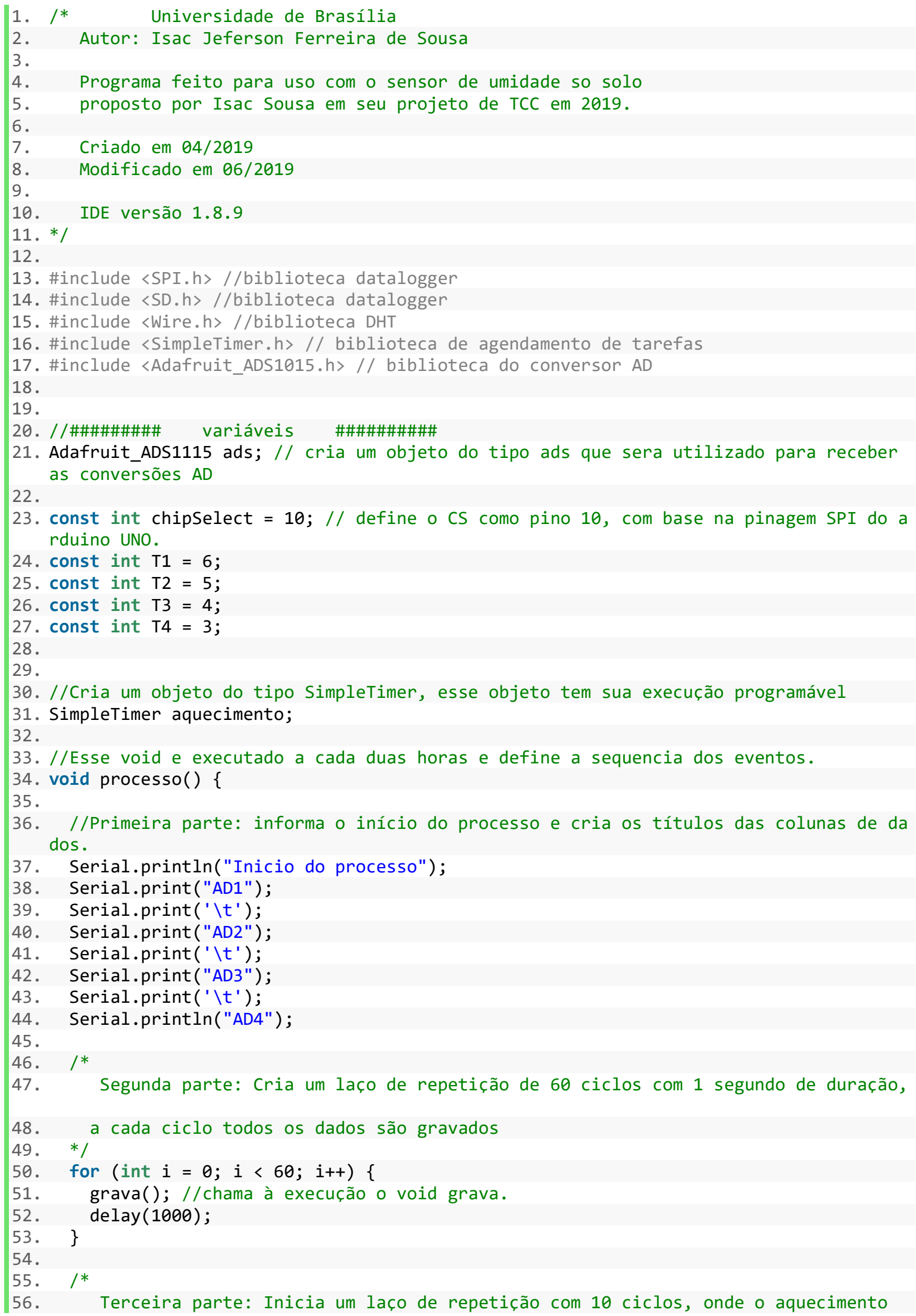


W).

e ligado $(\mathrm{HIGH})$, posteriormente ao fim desse laço o aquecimento e desligado (LO

58. */

59. Serial.println("Inicio do aquecimento");

60. for (int $\mathrm{e}=0$; e $<10$; e++) \{

61.

62.

63.

64.

65.

66.

67.

68. \}

digitalWrite(T4, HIGH);//define a saída como $5 \mathrm{~V}$

digitalWrite(T3, HIGH);

digitalWrite(T2, HIGH);

digitalWrite(T1, HIGH);

grava();

\}

delay (1000);

Serial.println("Fim do aquecimento");

digitalWrite(T4, LOW);//define a saída como OV

digitalWrite(T3, LOW);

digitalWrite(T2, LOW);

digitalWrite(T1, LOW);

/* $* /$

Quarta parte: Um laço com 600 ciclos para registrar o resfriamento

for (int $u=0 ; u<600 ; u++$ ) \{

grava();

\} delay (1000);

82. Serial.println("Fim do processo");

83. \}//fim do void processo

84.

85. void grava() \{

86 .

87. //define as variáveis que vão receber os valores digitais

88. int16_t adc0, adc1, adc2, adc3;

89.

90.

91.

92.

93.

94.

95.

96.

97.

98.

99.

100.

101.

102.

103.

104.

105.

106.

107.

108.

109.

110.

111.

112.

113.

114.

115.

116.

117.

118.

119.

//localiza e distribui os valores obtidos pelo ADS1115

$\operatorname{adc} \theta=$ ads.readADC_SingleEnded $(0)$;

adc1 = ads.readADC_SingleEnded $(1)$;

adc2 = ads.readADC_SingleEnded $(2)$;

adc3 = ads.readADC_SingleEnded $(3)$;

//Cria uma string onde serão adicionados os valores que serão gravados.

String datastring = " "

datastring $+=$ ('\} t ^ { \prime } ) \text { ; }

datastring $+=$ adco;

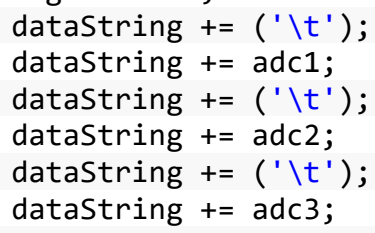


121.

122. void $\operatorname{setup}()\{$

123.

124. Serial.begin(57600);

125. //define os pinos que controlam os transistores como saída

126. $\quad \operatorname{pinMode}(\mathrm{T} 1$, OUTPUT);

127. pinMode(T2, OUTPUT);

128. $\quad$ pinMode(T3, OUTPUT);

129. pinMode(T4, OUTPUT);

130. //Inicia os pinos que controlam a saida como OV

131. digitalWrite(T1, LOW);

132. digitalWrite(T2, LOW);

133. digitalWrite(T3, LOW);

134. digitalWrite(T4, LOW);

135. ads.begin(); // dá início ao conversor AD

136.

137. ervalo de tempo

//Define qual processo deve ser realizado pelo SimpleTimer assim como o int 138.

139.

140.

141.

142.

143.

144.

145 .

aquecimento.setInterval(7200000, processo);

\}// fim do void setup

void loop() \{

aquecimento.run(); //aciona a contagem do objeto SimpleTimer

grava( );

delay (600000);

146. \} // fim do void loop 